\title{
ON A QUADRATIC WARING'S PROBLEM WITH CONGRUENCE CONDITIONS
}

\author{
DAEJUN KIM
}

\begin{abstract}
For each positive integer $n$, let $g_{\Delta}(n)$ be the smallest positive integer $g$ such that every complete quadratic polynomial in $n$ variables which can be represented by a sum of odd squares is represented by a sum of at most $g$ odd squares. In this paper, we analyze $g_{\Delta}(n)$ by studying representations of integral quadratic forms by sums of squares with certain congruence condition. We prove that the growth of $g_{\Delta}(n)$ is at most an exponential of $\sqrt{n}$, which is the same as the best known upper bound on the $g$-invariants of the original quadratic Waring's problem. We also determine the exact value of $g_{\Delta}(n)$ for each positive integer less than or equal to 4 .
\end{abstract}

\section{INTRODUCTION}

In 1770, Lagrange proved the Four-Square Theorem, which states that every positive integer is a sum of at most four squares of integers. This result has been generalized in many directions. In 1930's, a higher dimensional generalization, the so-called new (or quadratic) Waring's Problem, was initiated and studied by Mordell $12]$ and Ko [10]. In those papers, they proved that for any integer $1 \leq n \leq 5$, every positive definite integral quadratic form in $n$ variables is represented by a sum of $n+3$ squares, and $n+3$ is the smallest number with this property. Later, Mordell [13] proved that the quadratic form corresponding to the root lattice $E_{6}$ cannot be represented by any sum of squares.

This result of Mordell lead us to consider the number $g_{\mathbb{Z}}(n)$ defined as the smallest positive integer $g$ such that every quadratic form in $n$ variables which can be represented by a sum of squares is represented by a sum of at most $g$ squares. The numbers $g_{\mathbb{Z}}(n)$ are called the " $g$-invariants" of $\mathbb{Z}$. Then the results of Lagrange's, Mordell's and Ko's mentioned above can now be rewritten as $g_{\mathbb{Z}}(n)=n+3$ for $1 \leq n \leq 5$. In [7, Kim and Oh proved that $g_{\mathbb{Z}}(6)=10$, which disproves the earlier conjecture made by Ko [11] that $g_{\mathbb{Z}}(6)=9$. This is the last known value of $g_{\mathbb{Z}}(n)$.

On the other hand, it has been studied to find an upper bound of $g_{\mathbb{Z}}(n)$ as a function of $n$. Icaza [5 gave the first explicit but astronomical upper bound by computing the so called HKK-constant in [4]. Later, Kim-Oh [8] proved that $g_{\mathbb{Z}}(n)=O\left(3^{n / 2} n \log n\right)$, which improves Icaza's bound. Recently, Beli-Chan-IcazaLiu [1] obtained a better upper bound $g_{\mathbb{Z}}(n)=O\left(e^{(4+2 \sqrt{2}+\varepsilon) \sqrt{n}}\right)$ for any $\varepsilon>0$.

In this paper, we consider a quadratic Waring's problem with a congruence condition modulo 2 as a generalization of the original problem. One may naturally generalize the Lagrange's four square theorem by considering the smallest number

2010 Mathematics Subject Classification. Primary 11E12, 11E25.

Key words and phrases. Waring's problem, Sums of squares, Representations of cosets.

This work was supported by BK21 PLUS SNU Mathematical Science Division. 
$r$ such that every positive integer is a sum of at most $r$ squares of odd integers. In fact, this number is 10 and

$$
42=5^{2}+3^{2}+1^{2}+1^{2}+1^{2}+1^{2}+1^{2}+1^{2}+1^{2}+1^{2}
$$

is the smallest positive integer which is a sum of 10 squares of odd integers but is not a sum of less than 10 squares of odd integers (cf. Proposition 3.4 see also [6]).

As a higher dimensional generalization of this problem, we introduce new $g$ invariants $g_{\Delta}(n)$ in the following paragraphs.

Let $f(\mathbf{x})=f\left(x_{1}, \ldots, x_{n}\right)$ be a quadratic polynomial such that

$$
f(\mathbf{x})=Q(\mathbf{x})+L(\mathbf{x})+c,
$$

where $Q(\mathbf{x})$ is a quadratic form, $L(\mathbf{x})$ is a linear form, and $c$ is a constant. We always assume that $Q$ is positive definite. Hence, there exists a unique vector $\mathbf{w}_{f} \in \mathbb{Q}^{n}$ such that $L(\mathbf{x})=2 B\left(\mathbf{x}, \mathbf{w}_{f}\right)$, where $B$ is the bilinear form such that $B(\mathbf{x}, \mathbf{x})=Q(\mathbf{x})$. The quadratic polynomial $f(\mathbf{x})$ is called complete if $c=Q\left(\mathbf{w}_{f}\right)$, that is,

$$
f(\mathbf{x})=Q(\mathbf{x})+2 B\left(\mathbf{x}, \mathbf{w}_{f}\right)+Q\left(\mathbf{w}_{f}\right)=Q\left(\mathbf{x}+\mathbf{w}_{f}\right) .
$$

We say that a quadratic polynomial $f(\mathbf{x})=f\left(x_{1}, \ldots, x_{n}\right)$ is represented by a quadratic polynomial $g(\mathbf{y})=g\left(y_{1}, \ldots, y_{m}\right)(m \geq n)$ if there exists $T \in M_{n \times m}(\mathbb{Z})$ and $\mathbf{c} \in \mathbb{Z}^{n}$ such that

$$
f(\mathbf{x})=g(\mathbf{x} T+\mathbf{c}) .
$$

Now let $\Delta_{r}(\mathbf{y})$ be the following quadratic polynomial in variables $\mathbf{y}=\left(y_{1}, \ldots, y_{r}\right)$ :

$$
\Delta_{r}\left(y_{1}, \ldots, y_{r}\right):=\left(2 y_{1}+1\right)^{2}+\cdots+\left(2 y_{r}+1\right)^{2} .
$$

A quadratic polynomial $f(\mathbf{x})$ is said to be represented by a sum of $r$ odd squares if it is represented by $\Delta_{r}(\mathbf{y})$. For each positive integer $n$, we define the set $\mathcal{F}_{n}$ of all complete quadratic polynomials $f(\mathbf{x})$ in variables $\mathbf{x}=\left(x_{1}, \ldots, x_{n}\right)$ which can be represented by a sum of odd squares. For a quadratic polynomial $f(\mathbf{x})$ in $\mathcal{F}_{n}$, we define

$$
r(f):=\min \left\{r \in \mathbb{N}: f(\mathbf{x}) \text { can be represented by } \Delta_{r}(\mathbf{y})\right\},
$$

and we define the following new $g$-invariant of $\Delta_{r}$ :

$$
g_{\Delta}(n):=\max \left\{r(f): f(\mathbf{x}) \in \mathcal{F}_{n}\right\} .
$$

One may deduce that the problem of determining $g_{\Delta}(1)$ is equivalent to the problem of representing positive integers by sums of odd squares explained above, so that $g_{\Delta}(1)=10$. Furthermore, we will see in Section 3 that $g_{\Delta}(n)$ can be analyzed by studying representation of integral quadratic forms by sums of squares with a congruence condition modulo 2. Our main results can be stated as

Theorem 1.1. Let $n$ be a positive integer. For any $\varepsilon>0$ we have

$$
g_{\Delta}(n)=O\left(e^{(4+2 \sqrt{2}+\varepsilon) \sqrt{n}}\right) .
$$

Theorem 1.2. We have $g_{\Delta}(1)=10$ and $g_{\Delta}(n)=n+10$ for $n=2,3,4$.

Note that our result presents the same growth as the best known upper bound on $g_{\mathbb{Z}}(n)$. More precisely, the upper bound on $g_{\Delta}(n)$ we obtain is approximately $n^{2}$ times the upper bound on $g_{\mathbb{Z}}(n)$ obtained in [1]. We will adopt geometric language of quadratic spaces, lattices and $\mathbb{Z}$-cosets in studying $g_{\Delta}(n)$ so that we shall use the geometric theory of those. 
The rest of the paper is organized as follows. In Section 2 we introduce the geometric language and theory of quadratic spaces, lattices and $\mathbb{Z}$-cosets, especially the concept of representations of $\mathbb{Z}$-cosets. In Section 3 , we consider the problem geometrically by translating representations of quadratic polynomials into representations of $\mathbb{Z}$-cosets explicitly. The exact value of $g_{\Delta}(1)$ will also be determined. Section 4 contains some technical lemmas which will essentially be used in the following sections. The proof of Theorem 1.1 will be presented in Section [5. In Section [6, we will determine the exact values of $g_{\Delta}(n)$ for $n=2,3,4$ through some extensive computation.

For any unexplained notations, terminologies, and basic facts about $\mathbb{Z}$-lattices, we refer the readers to [15].

\section{REPRESEntation of COSEtS}

In this section, we introduce the geometric theory of quadratic $\mathbb{Z}$-lattices. We refer the readers to [3, Section 4] for the theory under more general setting. For simplicity, the quadratic map and its associated bilinear form on any quadratic space will be denoted by $Q$ and $B$, respectively. The set of all places on $\mathbb{Q}$ including the infinite place $\infty$ will be denoted by $\Omega$.

A $\mathbb{Z}$-lattice is a finitely generated $\mathbb{Z}$-module (hence a free $\mathbb{Z}$-module) $L$ on an $m$-dimensional quadratic space $V$ over $\mathbb{Q}$. A $\mathbb{Z}$-coset is a set $L+\mathbf{v}$, where $L$ is a $\mathbb{Z}$ lattice on $V$ and $\mathbf{v}$ is a vector in $V$. A $\mathbb{Z}$-coset $K+\mathbf{w}$ on an $n$-dimensional quadratic space $W$ is said to be represented by another $\mathbb{Z}$-coset $L+\mathbf{v}$ on an $m$-dimensional space $V$, which is denoted by

$$
K+\mathbf{w} \rightarrow L+\mathbf{v}
$$

if there exists an isometry $\sigma: W \rightarrow V$ such that $\sigma(K+\mathbf{w}) \subseteq L+\mathbf{v}$, which is equivalent to

$$
\sigma(K) \subseteq L \quad \text { and } \quad \sigma(\mathbf{w})-\mathbf{v} \in L .
$$

Two $\mathbb{Z}$-cosets $K+\mathbf{w}$ and $L+\mathbf{v}$ are said to be isometric, which is denoted by $K+\mathbf{w} \cong L+\mathbf{v}$, if one is represented by another one and vice versa. For each $p \in \Omega-\{\infty\}, \mathbb{Z}_{p}$-cosets and representations of $\mathbb{Z}_{p}$-cosets are defined analogously.

As in the case of quadratic forms and lattices, there is a one-to-one correspondence between the set of equivalence classes of complete quadratic polynomials in $n$ variables and the set of isometry classes of $\mathbb{Z}$-cosets on $n$-dimensional quadratic spaces. We will describe this correspondence concretely in Proposition 3.1 .

Definition 2.1. Let $L+\mathbf{v}$ be a $\mathbb{Z}$-coset on a quadratic space $V$. The genus of $L+\mathbf{v}$ is the set

$$
\operatorname{gen}(L+\mathbf{v})=\left\{K+\mathbf{w} \text { on } V: K_{p}+\mathbf{w} \cong L_{p}+\mathbf{v} \text { for any } p \in \Omega\right\} .
$$

Lemma 2.2. Let $L+\mathbf{v}$ be a $\mathbb{Z}$-coset on a quadratic space $V$ and let $S$ be a finite subset of $\Omega$. Suppose that $\mathbb{Z}_{p}$-coset $L(p)+\mathbf{x}_{p}$ on $V_{p}$ is given for each $p \in S$. Then there exists a $\mathbb{Z}$-coset $M+\mathbf{z}$ on $V$ such that

$$
M_{p}+\mathbf{z}= \begin{cases}L(p)+\mathbf{x}_{p} & \text { if } p \in S, \\ L_{p}+\mathbf{v} & \text { if } p \in \Omega-S .\end{cases}
$$

Proof. See Lemma 4.2 of 3 ]. 
Let $O_{\mathbb{A}}(V)$ be the adelization of the orthogonal group of $V$. By Lemma 2.2 $O_{\mathbb{A}}(V)$ naturally acts transitively on $\operatorname{gen}(L+\mathbf{v})$ and hence

$$
\operatorname{gen}(L+\mathbf{v})=O_{\mathbb{A}}(V) \cdot(L+\mathbf{v}) .
$$

Let $O_{\mathbb{A}}(L+\mathbf{v})$ be the stabilizer of $L+\mathbf{v}$ in $O_{\mathbb{A}}(V)$. Then the isometry classes in gen $(L+\mathbf{v})$ can be identified with

$$
O(V) \backslash O_{\mathbb{A}}(V) / O_{\mathbb{A}}(L+\mathbf{v}) .
$$

The class number of $L+\mathbf{v}$, denoted by $h(L+\mathbf{v})$, is the number of classes in $\operatorname{gen}(L+\mathbf{v})$, which is also the number of elements in $O(V) \backslash O_{\mathbb{A}}(V) / O_{\mathbb{A}}(L+\mathbf{v})$. The class number $h(L+\mathbf{v})$ is finite and $h(L+\mathbf{v}) \geq h(L)$, where $h(L)$ is the class number of $L$ (see Corollary 4.4 of [3]). Note that $h(L)$ is equal to the number of elements in $O(V) \backslash O_{\mathbb{A}}(V) / O_{\mathbb{A}}(L)$. For each $p \in \Omega$, we have

$$
O\left(L_{p}+\mathbf{v}\right)=\left\{\sigma \in O\left(V_{p}\right): \sigma\left(L_{p}\right)=L_{p} \text { and } \sigma(\mathbf{v}) \equiv \mathbf{v} \bmod L_{p}\right\} \subseteq O\left(L_{p}\right) .
$$

From now on, let $I_{n}=\mathbb{Z}\left[\mathbf{e}_{1}, \ldots, \mathbf{e}_{n}\right]$ be the $\mathbb{Z}$-lattice whose Gram matrix with respect to $\left\{\mathbf{e}_{1}, \ldots, \mathbf{e}_{n}\right\}$ is the identity matrix. For the sake of convenience, the vector $\mathbf{e}_{1}+\cdots+\mathbf{e}_{n}$ will be denoted by $\mathbf{v}_{n}$ and the $\mathbb{Z}$-coset $I_{n}+\frac{1}{2} \mathbf{v}_{n}$ will be denoted by $\Sigma_{n}$.

Proposition 2.3. For any $1 \leq n \leq 8$, we have $h\left(\Sigma_{n}\right)=1$.

Proof. If we can prove for any prime $p$ that

$$
O\left(\left(\Sigma_{n}\right)_{p}\right)=O\left(\left(I_{n}\right)_{p}\right)
$$

then $O_{\mathbb{A}}\left(\Sigma_{n}\right)=O_{\mathbb{A}}\left(I_{n}\right)$ so that we have $h\left(\Sigma_{n}\right)=h\left(I_{n}\right)=1$, which proves the proposition.

When $p \neq 2$, we have $\left(\Sigma_{n}\right)_{p}=\left(I_{n}\right)_{p}+\frac{1}{2} \mathbf{v}_{n}=\left(I_{n}\right)_{p}$ so that $O\left(\left(\Sigma_{n}\right)_{p}\right)=O\left(\left(I_{n}\right)_{p}\right)$. Now, it suffices to show that $O\left(\left(I_{n}\right)_{2}\right) \subseteq O\left(\left(\Sigma_{n}\right)_{2}\right)$. Let $\sigma \in O\left(\left(I_{n}\right)_{2}\right)$ and for each $1 \leq i \leq n$, we put $\sigma\left(\mathbf{e}_{i}\right)=\sum_{k=1}^{n} t_{i k} \mathbf{e}_{k}$ for some $t_{i k} \in \mathbb{Z}_{2}$. Note that

$$
\sum_{k=1}^{n} t_{i k}^{2}=Q\left(\sigma\left(\mathbf{e}_{i}\right)\right)=1 \quad \text { and } \quad \sum_{k=1}^{n} t_{i k} t_{j k}=B\left(\sigma\left(\mathbf{e}_{i}\right), \sigma\left(\mathbf{e}_{j}\right)\right)=0,
$$

for any $1 \leq i \neq j \leq n$, since $\sigma \in O\left(\left(I_{n}\right)_{2}\right)$. We claim that $\sum_{i=1}^{n} t_{i k} \in 1+2 \mathbb{Z}_{2}$ for any $1 \leq k \leq n$ if the following two conditions hold:

(1) $\sum_{k=1}^{n} t_{i k}^{2} \in 1+4 \mathbb{Z}_{2}$ for any $1 \leq i \leq n$,

(2) $\sum_{k=1}^{n} t_{i k} t_{j k} \in 2 \mathbb{Z}_{2}$ for any $1 \leq i \neq j \leq n$.

If we show this claim, then we have

$$
\sigma\left(\frac{1}{2} \mathbf{v}_{n}\right)=\sum_{i=1}^{n} \frac{1}{2} \sigma\left(\mathbf{e}_{i}\right)=\sum_{k=1}^{n}\left(\frac{1}{2} \cdot \sum_{i=1}^{n} t_{i k}\right) \mathbf{e}_{k} \in\left(\Sigma_{n}\right)_{2},
$$

which implies $\sigma \in O\left(\left(\Sigma_{n}\right)_{2}\right)$ and therefore we prove the proposition.

We prove the claim using an induction argument on $n$. When $n=1$, we have $t_{11}= \pm 1 \in 1+2 \mathbb{Z}_{2}$. Now, assume that $n>1$ and the above two conditions hold. From the first condition, for each $1 \leq i \leq n$, exactly one or five of $\left\{t_{i 1}, \ldots, t_{i n}\right\}$ belong to $1+2 \mathbb{Z}_{2}$ and all the other elements are in $2 \mathbb{Z}_{2}$. Assume, without loss of generality, that $t_{n n} \in 1+2 \mathbb{Z}_{2}$ and $t_{n k} \in 2 \mathbb{Z}_{2}$ for all $k<n$. Then, from the second condition, $t_{i n} \in 2 \mathbb{Z}_{2}$ for any $i<n$. Hence, we have $\sum_{i=1}^{n} t_{i n} \in 1+2 \mathbb{Z}_{2}$. Also, we have $\sum_{k=1}^{n-1} t_{i k}^{2} \in 1+4 \mathbb{Z}_{2}$ and $\sum_{k=1}^{n-1} t_{i k} t_{j k} \in 2 \mathbb{Z}_{2}$ for any $1 \leq i \neq j \leq n-1$ so that we have $\sum_{i=1}^{n} t_{i k} \in 1+2 \mathbb{Z}_{2}$ by the induction hypothesis. Therefore, we are 
left with the case when $n \geq 5$ and exactly five of $\left\{t_{i 1}, \ldots, t_{i n}\right\}$ belong to $1+2 \mathbb{Z}_{2}$ for any $1 \leq i \leq n$. One may easily show from the second condition that this can only happen when $n=6,8$ as well as $\sum_{i=1}^{n} t_{i k} \in 1+2 \mathbb{Z}_{2}$ for any $1 \leq k \leq n$ in those cases.

Proposition 2.4. Let $K+\mathbf{w}$ be a $\mathbb{Z}$-coset on a quadratic space $W$, and let $L+\mathbf{v}$ be a $\mathbb{Z}$-coset on a quadratic space $V$. Suppose that for each $p \in \Omega$, there exists a representation $\sigma_{p}: W_{p} \rightarrow V_{p}$ such that $\sigma_{p}\left(K_{p}+\mathbf{w}\right) \subseteq L_{p}+\mathbf{v}$. Then there exists a $\mathbb{Z}$-coset $M+\mathbf{z} \in \operatorname{gen}(L+\mathbf{v})$ which represents $K+\mathbf{w}$.

Proof. By virtue of the Hasse Principle, we may assume that $W \subseteq V$. By Witt's extension theorem, we may further assume that $\sigma_{p} \in O\left(V_{p}\right)$. Let $S$ be the set of $p \in \Omega$ such that $K_{p}+\mathbf{w} \nsubseteq L_{p}+\mathbf{v}$. Then $S$ is a finite set since $K_{p}+\mathbf{w}=K_{p} \subseteq$ $L_{p}=L_{p}+\mathbf{v}$ for almost all $p$. For each $p \in S$, let $L(p)=\sigma_{p}^{-1}\left(L_{p}\right)$ and $\mathbf{x}_{p}=\sigma_{p}^{-1}(\mathbf{v})$. By Lemma 2.2. there exist $M+\mathbf{z} \in \operatorname{gen}(L+\mathbf{v})$ such that

$$
M_{p}+\mathbf{z}= \begin{cases}\sigma_{p}^{-1}\left(L_{p}+\mathbf{v}\right) & \text { if } p \in S, \\ L_{p}+\mathbf{v} & \text { if } p \in \Omega-S .\end{cases}
$$

Therefore $K+\mathbf{w} \subseteq M+\mathbf{z}$, which proves the proposition.

Corollary 2.5. Let $K+\mathbf{w}$ be a $\mathbb{Z}$-coset and let $n$ be a positive integer less than or equal to 8 . If $K+\mathbf{w}$ is locally represented by $\Sigma_{n}$, then $K+\mathbf{w}$ is represented by $\Sigma_{n}$.

Proof. This is a direct consequence of Propositions 2.3 and 2.4

\section{Geometric approach of the problem}

In this section, we introduce some geometric approach of the problem via representations of $\mathbb{Z}$-cosets. For any $r \in \mathbb{N}$, let $I_{r}=\mathbb{Z}\left[\mathbf{e}_{1}, \ldots, \mathbf{e}_{r}\right]$ be the $\mathbb{Z}$-lattice whose Gram matrix with respect to $\left\{\mathbf{e}_{1}, \ldots, \mathbf{e}_{r}\right\}$ is the identity matrix. As in Section 2, the vector $\mathbf{e}_{1}+\cdots+\mathbf{e}_{r}$ will be denoted by $\mathbf{v}_{r}$ and the $\mathbb{Z}$-coset $I_{r}+\frac{1}{2} \mathbf{v}_{r}$ will be denoted by $\Sigma_{r}$. For any positive integer $n$, we define

$$
\mathcal{K}_{n}:=\left\{K+\mathbf{w} \mid \operatorname{rank}(K)=n, \mathbf{w} \in \mathbb{Q} K, \exists \sigma: K+\mathbf{w} \rightarrow \Sigma_{r}\right\} .
$$

For any $K+\mathbf{w} \in \mathcal{K}_{n}$, we define

$$
g(K+\mathbf{w}):=\min \left\{r \in \mathbb{N} \mid \exists \sigma: K+\mathbf{w} \rightarrow \Sigma_{r}\right\},
$$

and we also define

$$
g_{\Delta}^{\prime}(n):=\max \left\{g(K+\mathbf{w}) \mid K+\mathbf{w} \in \mathcal{K}_{n}\right\} .
$$

Proposition 3.1. For any positive integer $n$, we have $g_{\Delta}(n)=g_{\Delta}^{\prime}(n)$

Proof. Let $f(\mathbf{x})=Q\left(\mathbf{x}+\mathbf{w}_{f}\right)=\left(\mathbf{x}+\mathbf{w}_{f}\right) 4 M\left(\mathbf{x}+\mathbf{w}_{f}\right)^{t}$ be a complete quadratic polynomial in $\mathcal{F}_{n}$, where $\mathbf{w}_{f}=\left(w_{1}, \ldots, w_{n}\right) \in \mathbb{Q}^{n}$, and $4 M$ is the Gram matrix of the quadratic part $Q$ of $f$. Hence, there exists a positive integer $r:=r(f)$, a matrix $T=\left(t_{i j}\right) \in M_{n \times r}(\mathbb{Z})$, and a vector $\mathbf{c}=\left(c_{1}, \ldots, c_{n}\right) \in \mathbb{Z}^{n}$ such that

$$
\left(\mathbf{x}+\mathbf{w}_{f}\right)(4 M)\left(\mathbf{x}+\mathbf{w}_{f}\right)^{t}=f(\mathbf{x})=\Delta_{r}(\mathbf{x} T+\mathbf{c}) .
$$

By comparing the coefficients of both sides and by putting $\mathbf{x}=-\mathbf{w}_{f}$, one may easily show that

$$
M=T \cdot T^{t} \text { and }-\mathbf{w}_{f} T+\mathbf{c}+\frac{1}{2}(1, \ldots, 1)=\mathbf{0} \in \mathbb{Q}^{r} .
$$


Now let us consider a $\mathbb{Z}$-coset $K+\mathbf{w}$ of a $\mathbb{Z}$-lattice $K=\mathbb{Z}\left[\mathbf{d}_{1}, \ldots, \mathbf{d}_{n}\right] \cong M$, where $\mathbf{w}=w_{1} \mathbf{d}_{1}+\cdots+w_{n} \mathbf{d}_{n}$ and define a linear map $\sigma: K \rightarrow I_{r}$ by

$$
\sigma\left(\mathbf{d}_{i}\right)=\sum_{j=1}^{r} t_{i j} \mathbf{e}_{j} .
$$

By (3.1), the map $\sigma$ is a representation of $\mathbb{Z}$-lattices satisfying $\sigma(\mathbf{w})-\frac{1}{2} \mathbf{v}_{r} \in I_{r}$, which implies that $\sigma: K+\mathbf{w} \rightarrow \Sigma_{r}$ is a representation of $\mathbb{Z}$-cosets. Thus, we have constructed a $\mathbb{Z}$-coset $K+\mathbf{w}$ in $\mathcal{K}_{n}$ with $g(K+\mathbf{w}) \leq r=r(f)$.

Conversely, let $K+\mathbf{w}$ be a $\mathbb{Z}$-coset in $\mathcal{K}_{n}$, where $K=\mathbb{Z}\left[\mathbf{d}_{1}, \ldots, \mathbf{d}_{n}\right]$ and $\mathbf{w}=$ $w_{1} \mathbf{d}_{1}+\cdots+w_{n} \mathbf{d}_{n}$. Then there exist $g:=g(K+\mathbf{w}) \in \mathbb{N}$ and a representation of $\mathbb{Z}$-cosets $\sigma: K+\mathbf{w} \rightarrow \Sigma_{g}$. Since $\sigma(\mathbf{w})-\frac{1}{2} \mathbf{v}_{g} \in I_{g}$, there are integers $c_{1}, \ldots, c_{g}$ such that $\sigma(\mathbf{w})=\frac{1}{2} \mathbf{v}_{g}+c_{1} \mathbf{e}_{1}+\cdots+c_{g} \mathbf{e}_{g}$. Also, let $T=\left(t_{i j}\right) \in M_{n \times g}(\mathbb{Z})$ be the matrix such that $\sigma\left(\mathbf{d}_{i}\right)=\sum_{j=1}^{g} t_{i j} \mathbf{e}_{j}$ for each $1 \leq i \leq n$. Then we have

$$
\begin{aligned}
f(\mathbf{x}) & :=4 \cdot Q\left(x_{1} \mathbf{d}_{1}+\cdots+x_{n} \mathbf{d}_{n}+\mathbf{w}\right) \\
& =4 \cdot Q\left(\sigma\left(x_{1} \mathbf{d}_{1}+\cdots+x_{n} \mathbf{d}_{n}+\mathbf{w}\right)\right) \\
& =4 \cdot Q\left(\left[\left(x_{1}, \ldots, x_{n}\right) T+\left(c_{1}, \ldots, c_{g}\right)\right] \cdot\left(\mathbf{e}_{1}, \ldots, \mathbf{e}_{g}\right)^{t}+\frac{1}{2} \mathbf{v}_{g}\right) \\
& =\Delta_{g}\left(\left(x_{1}, \ldots, x_{n}\right) T+\left(c_{1}, \ldots, c_{g}\right)\right),
\end{aligned}
$$

where $\mathbf{x}=\left(x_{1}, \ldots, x_{n}\right)$. Hence the complete quadratic polynomial $f(\mathbf{x})$ is represented by $\Delta_{g}(\mathbf{y})$. Therefore, we have constructed a quadratic polynomial $f(\mathbf{x})$ in $\mathcal{F}_{n}$ with $r(f) \leq g=g(K+\mathbf{w})$. The proposition follows as a consequence.

Proposition 3.2. For any positive integer n, let

$$
\mathcal{K}_{n}^{*}:=\left\{K+\mathbf{w} \in \mathcal{K}_{n} \mid \mathbf{w}=\frac{1}{2} \mathbf{k}, \mathbf{k} \text { is a primitive vector of } K\right\} .
$$

Then we have

$$
g_{\Delta}(n)=\max \left\{g(K+\mathbf{w}) \mid K+\mathbf{w} \in \mathcal{K}_{n}^{*}\right\} .
$$

Proof. Let $K+\mathbf{w}$ be a $\mathbb{Z}$-coset in $\mathcal{K}_{n}$ and let $\sigma: K+\mathbf{w} \rightarrow \Sigma_{g}$ be a representation of $\mathbb{Z}$-cosets, where $g=g(K+\mathbf{w})$. Note that one can write $\mathbf{w}=\frac{d}{m} \mathbf{k}$, where $d, m$ are relatively prime positive integers and $\mathbf{k}$ is a primitive vector of $K$. Since $\sigma(K) \subseteq I_{g}$, there are integers $a_{1}, \ldots, a_{g}$ such that $\sigma(\mathbf{k})=a_{1} \mathbf{e}_{1}+\cdots+a_{g} \mathbf{e}_{g}$. Moreover, we have

$$
\sigma\left(\frac{d}{m} \mathbf{k}\right)-\frac{1}{2} \mathbf{v}_{g}=\sum_{j=1}^{g}\left(\frac{d}{m} a_{j}-\frac{1}{2}\right) \mathbf{e}_{j} \in I_{g} .
$$

Therefore, we have $\frac{d}{m} a_{j}-\frac{1}{2} \in \mathbb{Z}$, that is, $d a_{j}-\frac{m}{2} \in m \mathbb{Z}$ for any $1 \leq j \leq g$. Hence, there is an positive integer $m_{0}$ such that $m=2 m_{0}$ and we have

$$
d \equiv 1(\bmod 2) \quad \text { and } \quad a_{j} \equiv 0\left(\bmod m_{0}\right) \text { for any } 1 \leq j \leq g .
$$

Thus, $Q(\mathbf{k}) \in m_{0}^{2} \mathbb{Z}$ and $B(\mathbf{k}, K) \subseteq m_{0} \mathbb{Z}$.

Since $\mathbf{k}$ is a primitive vector of $K$, we may assume that $K=\mathbb{Z}\left[\mathbf{k}, \mathbf{k}_{2}, \ldots, \mathbf{k}_{n}\right]$ and consider the $\mathbb{Z}$-lattice $\tilde{K}=\mathbb{Z}\left[\frac{\mathbf{k}}{m_{0}}, \mathbf{k}_{2}, \ldots, \mathbf{k}_{n}\right]$ in the same quadratic space $\mathbb{Q} K$. Note that $\frac{\mathbf{k}}{m_{0}}$ is a primitive vector of $\tilde{K}$. One may easily check that

$$
\sigma(\tilde{K}) \subseteq I_{g} \quad \text { and } \quad \sigma\left(\frac{d}{2} \cdot \frac{\mathbf{k}}{m_{0}}\right)-\frac{1}{2} \mathbf{v}_{g} \in I_{g},
$$


which implies that $\sigma: \tilde{K}+\frac{d}{2}\left(\frac{\mathbf{k}}{m_{0}}\right) \rightarrow \Sigma_{g}$ is a representation of $\mathbb{Z}$-cosets. Therefore, we have

$$
\tilde{K}+\frac{d}{2} \cdot \frac{\mathbf{k}}{m_{0}}=\tilde{K}+\frac{1}{2} \cdot \frac{\mathbf{k}}{m_{0}} \in \mathcal{K}_{n}^{*} \quad \text { and } \quad g \geq \tilde{g}:=g\left(\tilde{K}+\frac{1}{2} \cdot \frac{\mathbf{k}}{m_{0}}\right) .
$$

On the other hand, if we let $\tilde{\sigma}: \tilde{K}+\frac{1}{2}\left(\frac{\mathbf{k}}{m_{0}}\right) \rightarrow \Sigma_{\tilde{g}}$ be a representation of $\mathbb{Z}$-cosets, then by restricting $\tilde{\sigma}$ on $K+\mathbf{w}$ we obtain a representation of $K+\mathbf{w}$ by $\Sigma_{\tilde{g}}$. Thus we may conclude $g \leq \tilde{g}$. Hence $g=\tilde{g}$, which proves the proposition.

Remark 3.3. (a) Let $K+\frac{1}{2} \mathbf{w}$ be a $\mathbb{Z}$-coset in $\mathcal{K}_{n}^{*}$, where $K=\mathbb{Z}\left[\mathbf{d}_{1}, \ldots, \mathbf{d}_{n}\right]$ and let $M$ be the Gram matrix corresponding to $K$ with respect to the basis $\left\{\mathbf{d}_{1}, \ldots, \mathbf{d}_{n}\right\}$. Then we may assume that $\mathbf{w}=\mathbf{d}_{i_{1}}+\cdots+\mathbf{d}_{i_{t}}$, where $1 \leq i_{1}<\cdots<i_{t} \leq n$ and $t \geq 1$. Let $R$ be either $\mathbb{Z}$ or $\mathbb{Z}_{2}$ and let us consider $K+\frac{1}{2} \mathbf{w}$ as an $R$-coset. Let $\sigma: K+\frac{1}{2} \mathbf{w} \rightarrow \Sigma_{r}$ be a representation of $R$-cosets, that is, $\sigma: K \rightarrow I_{r}$ and $\sigma\left(\frac{1}{2} \mathbf{w}\right)-\frac{1}{2} \mathbf{v}_{r} \in I_{r}$. Let $T=\left(t_{i j}\right)$ be the $n \times r$ matrix over $R$ such that $\sigma\left(\mathbf{d}_{i}\right)=\sum_{j=1}^{r} t_{i j} \mathbf{e}_{j}$ for any $1 \leq i \leq n$. Then the assumption that $\sigma$ is a representation of $R$-cosets is equivalent to the following conditions:

$$
M=T T^{t} \text { and } \sum_{i \in\left\{i_{1}, \ldots, i_{t}\right\}} t_{i j} \equiv 1(\bmod 2) \text { for each } 1 \leq j \leq r .
$$

Conversely, a matrix $T \in M_{n \times r}(R)$ satisfying (3.3) induces the representation of $R$-cosets $\sigma: K+\frac{1}{2} \mathbf{w} \rightarrow \Sigma_{r}$ defined by $\sigma\left(\mathbf{d}_{i}\right)=\sum_{j=1}^{r} t_{i j} \mathbf{e}_{j}$ for each $1 \leq i \leq n$. Therefore, we shall identify the above $\sigma$ with $T$.

(b) Let $M$ be an $n \times n$ symmetric matrix over $R$, which is not necessarily nondegenerate. We will sometimes say $M+\frac{1}{2} \mathbf{w}$ is represented by $\Sigma_{r}$, denoted by $M+\frac{1}{2} \mathbf{w} \rightarrow \Sigma_{r}$, which means that there exists an $n \times r$ integral matrix $T$ which satisfies (3.3). Suppose that there are two symmetric matrices $M_{1}, M_{2}$ over $R$ such that

$$
M_{i}+\frac{1}{2} \mathbf{w} \rightarrow \Sigma_{r_{i}} \quad \text { for each } i=1,2 .
$$

If we let $T_{i}$ be the corresponding $n \times r_{i}$ integral matrix for each $i$, then the $n \times\left(r_{1}+r_{2}\right)$ matrix $T=\left(T_{1} T_{2}\right)$ together with the $n \times n$ matrix $M=M_{1}+M_{2}$ satisfies (3.3), hence we have $M+\frac{1}{2} \mathbf{w} \rightarrow \Sigma_{r_{1}+r_{2}}$.

We can simply analyze the problem in the case when $n=1$.

Proposition 3.4. We have $g_{\Delta}(1)=10$.

Proof. Let $K+\frac{1}{2} \mathbf{w} \in \mathcal{K}_{n}^{*}$. As described in Remark 3.3 (a), we may assume that $\mathbf{w}=\mathbf{d}$, where $K=\mathbb{Z}[\mathbf{d}] \cong\langle M\rangle$ for some positive integer $M$. Furthermore, finding a representation of $\mathbb{Z}$-cosets $\sigma: K+\frac{1}{2} \mathbf{d} \rightarrow \Sigma_{r}$ is equivalent to writing $M$ as a sum of $r$ squares of odd integers.

We shall prove that every positive integer $M$ is a sum of at most 10 squares of odd integers. Clearly, 1 and 2 are sum of 1 and 2 odd squares, respectively. Now, let us assume that $M \equiv k(\bmod 8)$ with $3 \leq k \leq 10$. Then, $M-(k-3) \equiv 3(\bmod 8)$ so that Legendre's three-square theorem implies that $M-(k-3)=t_{1}^{2}+t_{2}^{2}+t_{3}^{2}$ for some odd integers $t_{1}, t_{2}, t_{3}$. Since $k-3$ is a sum of $k-3$ squares of $1, M$ is a sum of $k$ odd squares. Thus $g_{\Delta}(1) \leq 10$. On the other hand, every positive integers $M \equiv 2(\bmod 8)$ which are not a sum of two squares, for example, the integer 42 , is a sum of 10 odd squares. This proves the proposition. 


\section{LEMmas}

In this section, we will introduce several lemmas. We use the notations described in Remark 3.3 (a), so for a $\mathbb{Z}$-coset $K+\frac{1}{2} \mathbf{w} \in \mathcal{K}_{n}^{*}$, we put $K=\mathbb{Z}\left[\mathbf{d}_{1}, \ldots, \mathbf{d}_{n}\right]$ and $\mathbf{w}=\mathbf{d}_{i_{1}}+\cdots+\mathbf{d}_{i_{t}}$, where $1 \leq i_{1}<\cdots<i_{t} \leq n$ and $t \geq 1$. We begin with finding some necessary condition of a $\mathbb{Z}$-coset $K+\frac{1}{2} \mathbf{w} \in \mathcal{K}_{n}^{*}$ to be represented by $\Sigma_{r}$.

Lemma 4.1. Let $K+\frac{1}{2} \mathbf{w}$ be a $\mathbb{Z}$-coset in $\mathcal{K}_{n}^{*}$. If $K+\frac{1}{2} \mathbf{w}$ is represented by $\Sigma_{r}$ for some positive integer $r$, then the following holds.

(i) $Q(\mathbf{k}) \equiv B(\mathbf{w}, \mathbf{k})(\bmod 2)$ for any $\mathbf{k} \in K$.

(ii) $r \equiv Q(\mathbf{w})(\bmod 8)$ and $r \leq Q(\mathbf{w})$.

(iii) $r \leq r_{K, \mathbf{w}}$, where $r_{K, \mathbf{w}}$ is the greatest positive integer satisfying

$$
r_{K, \mathbf{w}} \leq Q\left(\mathbf{d}_{i_{1}}\right)+\cdots+Q\left(\mathbf{d}_{i_{t}}\right) \text { and } r_{K, \mathbf{w}} \equiv Q(\mathbf{w})(\bmod 8) .
$$

Proof. Let $\sigma: K+\frac{1}{2} \mathbf{w} \rightarrow \Sigma_{r}$ be a representation of $\mathbb{Z}$-cosets and $T=\left(t_{i j}\right)$ be the $n \times r$ integral matrix satisfying $\sigma\left(\mathbf{d}_{i}\right)=\sum_{j=1}^{r} t_{i j} \mathbf{e}_{j}$. Then,

$$
Q\left(\mathbf{d}_{i}\right)=Q\left(\sigma\left(\mathbf{d}_{i}\right)\right)=\sum_{j=1}^{r} t_{i j}^{2} \equiv \sum_{j=1}^{r} t_{i j}=B\left(\mathbf{v}_{r}, \sigma\left(\mathbf{d}_{i}\right)\right) \equiv B\left(\mathbf{w}, \mathbf{d}_{i}\right)(\bmod 2),
$$

for each $1 \leq i \leq n$. Hence, for any $\mathbf{k}=\sum_{i=1}^{n} k_{i} \mathbf{d}_{i} \in K$, we have

$$
Q(\mathbf{k}) \equiv \sum_{i=1}^{n} k_{i}^{2} Q\left(\mathbf{d}_{i}\right) \equiv \sum_{i=1}^{n} k_{i} B\left(\mathbf{w}, \mathbf{d}_{i}\right)=B(\mathbf{w}, \mathbf{k})(\bmod 2) .
$$

Now, we note that $\sigma(\mathbf{w})=\sum_{j=1}^{r} t_{\mathbf{w}, j} \mathbf{e}_{j}$, where $t_{\mathbf{w}, j}=\sum_{i \in\left\{i_{1}, \ldots, i_{t}\right\}} t_{i j}$ is an odd integer by the second condition of (3.3), for each $1 \leq j \leq r$. Thus, we have

$$
Q(\mathbf{w})=Q(\sigma(\mathbf{w}))=\sum_{j=1}^{r} t_{\mathbf{w}, j}^{2},
$$

so that $Q(\mathbf{w}) \equiv r(\bmod 8)$ and $r \leq Q(\mathbf{w})$. On the other hand, since not all elements in $\left\{t_{i_{1} j}, \ldots, t_{i_{t} j}\right\}$ are zero for each $1 \leq j \leq r$, we have

$$
r \leq \sum_{j=1}^{r}\left(\sum_{i \in\left\{i_{1}, \ldots, i_{t}\right\}} t_{i j}^{2}\right)=\sum_{i \in\left\{i_{1}, \ldots, i_{t}\right\}}\left(\sum_{j=1}^{r} t_{i j}^{2}\right)=Q\left(\mathbf{d}_{i_{1}}\right)+\cdots+Q\left(\mathbf{d}_{i_{t}}\right) .
$$

Then, $r \leq r_{K, \mathbf{w}}$ follows from this with $r \equiv Q(\mathbf{w})(\bmod 8)$.

Lemma 4.2. Let $K$ be a $\mathbb{Z}_{2}$-lattice of rank $n$ and $\mathbf{w}$ be a primitive vector of $K$. Suppose that $K+\frac{1}{2} \mathbf{w}$ is represented by $\Sigma_{g^{\prime}}$ over $\mathbb{Z}_{2}$ for some positive integer $g^{\prime}$. Then we have $g^{\prime} \equiv Q(\mathbf{w})(\bmod 8)$. Furthermore, we have

$$
K+\frac{1}{2} \mathbf{w} \rightarrow \Sigma_{g}
$$

for any positive integer $g \equiv Q(\mathbf{w})(\bmod 8)$ satisfying

$$
g \geq \begin{cases}n+3 & \text { if } Q(\mathbf{w}) \not \equiv 0(\bmod 4), \\ n+4 & \text { if } Q(\mathbf{w}) \equiv 0(\bmod 4) .\end{cases}
$$


Proof. For the sake of simplicity of notation, all the lattices, cosets, representations and matrices in the proof of this lemma are considered to be defined over $\mathbb{Z}_{2}$. Moreover, the $\mathbb{Z}_{2}$-lattice $I_{r}+\mathbb{Z}_{2}\left[\frac{1}{2} \mathbf{v}_{r}\right]$ associate with $\Sigma_{r}$ will be denoted by $L_{r}$ for any positive integer $r$ during the proof of this lemma.

Assume that there is a representation $\sigma^{\prime}: K+\frac{1}{2} \mathbf{w} \rightarrow \Sigma_{g^{\prime}}$ of $\mathbb{Z}_{2}$-cosets. One may show that $Q(\mathbf{w}) \equiv g^{\prime}(\bmod 8)$ holds by a similar argument used in the proof of (ii) of Lemma 4.1 .

Now, we prove the second assertion. Since $\sigma^{\prime}\left(\frac{1}{2} \mathbf{w}\right) \in L_{g^{\prime}}$, the representation $\sigma^{\prime}$ can be extended to a representation of $\mathbb{Z}_{2}$-lattices

$$
\sigma^{\prime}: K+\mathbb{Z}_{2}\left[\frac{1}{2} \mathbf{w}\right] \rightarrow L_{g^{\prime}}
$$

We shall divide the proof into three cases.

First, suppose that $Q(\mathbf{w}) \equiv 1(\bmod 2)$. One may easily verify that

$$
2\left(K+\mathbb{Z}_{2}\left[\frac{1}{2} \mathbf{w}\right]\right) \cong\langle Q(\mathbf{w})\rangle \perp 2 N,
$$

for some integral $\mathbb{Z}_{2}$-lattice $N$ and

$$
2 L_{g} \cong \begin{cases}\langle g\rangle \perp 4(\mathbb{H} \perp \ldots \perp \mathbb{H}) & \text { if } g \equiv \pm 1(\bmod 8), \\ \langle g\rangle \perp 4(\mathbb{H} \perp \ldots \perp \mathbb{H} \perp \mathbb{A}) & \text { if } g \equiv \pm 3(\bmod 8),\end{cases}
$$

where $\mathbb{H} \cong\left(\begin{array}{ll}0 & 1 \\ 1 & 0\end{array}\right)$ and $\mathbb{A} \cong\left(\begin{array}{ll}2 & 1 \\ 1 & 2\end{array}\right)$. It follows from Theorem 3 of 14 that $N$ has no proper unimodular Jordan component since $2\left(K+\mathbb{Z}_{2}\left[\frac{1}{2} \mathbf{w}\right]\right) \rightarrow 2 L_{g^{\prime}}$. On the other hand, the same theorem also implies that if $N$ has no proper unimodular Jordan component, then we have $2\left(K+\mathbb{Z}_{2}\left[\frac{1}{2} \mathbf{w}\right]\right) \rightarrow 2 L_{g}$ for any integer $g \equiv Q(\mathbf{w})(\bmod 8)$ with $g \geq n+3$. Therefore, there is a representation

$$
\sigma: K+\mathbb{Z}_{2}\left[\frac{1}{2} \mathbf{w}\right] \rightarrow L_{g} .
$$

Let $\sigma\left(\frac{1}{2} \mathbf{w}\right)=a_{1} \frac{\mathbf{v}_{g}}{2}+a_{2} \mathbf{e}_{2}+\cdots+a_{g} \mathbf{e}_{g}$, where the $a_{i}$ 's are 2-adic integers. Since

$$
Q(\sigma(\mathbf{w}))=a_{1}^{2} g+4\left(a_{2}^{2}+\cdots+a_{g}^{2}+a_{1}\left(a_{2}+\cdots+a_{g}\right)\right) \equiv a_{1}^{2} g(\bmod 4),
$$

we have $g \equiv Q(\mathbf{w})=Q(\sigma(\mathbf{w})) \equiv a_{1}^{2} g(\bmod 4)$. Hence, we have

$$
a_{1} \equiv 1(\bmod 2) \quad \text { and } \sigma(\mathbf{w}) \equiv \mathbf{v}_{g}\left(\bmod 2 I_{g}\right) \text {. }
$$

Similarly, for any vector $\mathbf{k} \in K$, let $\sigma(\mathbf{k})=b_{1} \frac{\mathbf{v}_{g}}{2}+b_{2} \mathbf{e}_{2}+\cdots+b_{g} \mathbf{e}_{g}$, where the $b_{i}$ 's are 2-adic integers. Since $Q(K) \subseteq \mathbb{Z}_{2}$ and

$$
Q(\mathbf{k})=Q(\sigma(\mathbf{k})) \equiv \frac{1}{4} b_{1}^{2} g(\bmod 1),
$$

we have $b_{1} \equiv 0(\bmod 2)$ so $\sigma(\mathbf{k}) \in I_{g}$. Hence, we have $\sigma(K) \subset I_{g}$ which implies that $\sigma: K+\frac{1}{2} \mathbf{w} \rightarrow \Sigma_{g}$ is a representation of $\mathbb{Z}_{2}$-cosets.

Next, suppose that $Q(\mathbf{w}) \equiv 2(\bmod 4)$. One can verify that

$$
2\left(K+\mathbb{Z}_{2}\left[\frac{1}{2} \mathbf{w}\right]\right) \cong\langle Q(\mathbf{w})\rangle \perp 2 N \text { or }\langle Q(\mathbf{w})\rangle \perp\langle 2 \varepsilon\rangle \perp 2 N,
$$

where $\varepsilon \in \mathbb{Z}_{2}^{\times}, N$ is an integral $\mathbb{Z}_{2}$-lattice and

$$
2 L_{g} \cong \begin{cases}\langle 2,2\rangle \perp 4(\mathbb{H} \perp \ldots \perp \mathbb{H}) & \text { if } g \equiv 2(\bmod 8), \\ \langle 6,14\rangle \perp 4(\mathbb{H} \perp \ldots \perp \mathbb{H} \perp \mathbb{A}) & \text { if } g \equiv 6(\bmod 8) .\end{cases}
$$


In this case, $2\left(K+\mathbb{Z}_{2}\left[\frac{1}{2} \mathbf{w}\right]\right) \rightarrow 2 L_{g^{\prime}}$ implies that $N$ has no proper unimodular Jordan component and

$$
\varepsilon \in \begin{cases}1+4 \mathbb{Z}_{2} & \text { if } Q(\mathbf{w}) \equiv 2(\bmod 8), \\ 3+4 \mathbb{Z}_{2} & \text { if } Q(\mathbf{w}) \equiv 6(\bmod 8) .\end{cases}
$$

On the other hand, if the above conditions for both $N$ and $\varepsilon$ are satisfied, then we have $2\left(K+\mathbb{Z}_{2}\left[\frac{1}{2} \mathbf{w}\right]\right) \rightarrow 2 L_{g}$ for any $g \equiv Q(\mathbf{w})(\bmod 8)$ with $g \geq n+3$. Therefore, we have a representation of cosets $\sigma: K+\frac{1}{2} \mathbf{w} \rightarrow \Sigma_{g}$ by a similar reasoning to the case when $Q(\mathbf{w}) \equiv 1(\bmod 2)$.

Finally, suppose that $Q(\mathbf{w}) \equiv 0(\bmod 4)$. Since $\mathbf{w}$ is a primitive vector of $K$, we may take $\left\{\mathbf{w}, \mathbf{d}_{2}, \ldots, \mathbf{d}_{n}\right\}$ as a basis for $K$. Let $T^{\prime}=\left(t_{i j}^{\prime}\right)$ be the $n \times g^{\prime}$ matrix over $\mathbb{Z}_{2}$ corresponding to the representation $\sigma^{\prime}$, that is,

$$
\left(\sigma^{\prime}(\mathbf{w}), \sigma^{\prime}\left(\mathbf{d}_{2}\right), \ldots, \sigma^{\prime}\left(\mathbf{d}_{n}\right)\right)^{t}=T^{\prime} \cdot\left(\mathbf{e}_{1}, \ldots, \mathbf{e}_{g^{\prime}}\right)^{t} .
$$

Then $t_{1 j}^{\prime} \in 1+2 \mathbb{Z}_{2}$ for any $1 \leq j \leq g^{\prime}$ (see Remark 3.3 (a)). Now, we consider another $\mathbb{Z}_{2}$-lattice $\tilde{K}=\mathbb{Z}_{2}\left[\tilde{\mathbf{w}}, \tilde{\mathbf{d}}_{2}, \ldots, \tilde{\mathbf{d}}_{n}\right]$ whose Gram matrix with respect to the basis $\left\{\tilde{\mathbf{w}}, \tilde{\mathbf{d}}_{2}, \ldots, \tilde{\mathbf{d}}_{n}\right\}$ is $T^{\prime} \cdot\left(T^{\prime}\right)^{t}+a E_{11}$, where $E_{11}$ is the $n \times n$ matrix with 1 in the $(1,1)$ position and 0 elsewhere and an integer $a \in\{7,15\}$ is chosen to satisfy $d \tilde{K}_{2} \neq 0$. Then the $n \times\left(g^{\prime}+a\right)$ matrix $\tilde{T}$ defined by

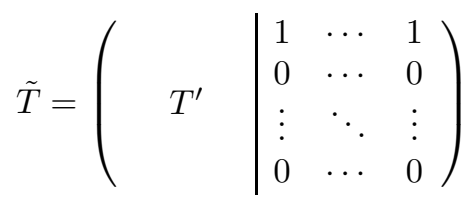

induces a representation of cosets $\tilde{\sigma}: \tilde{K}+\frac{1}{2} \tilde{\mathbf{w}} \rightarrow \Sigma_{g^{\prime}+a}$ (see Remark $\left.3.3(\mathrm{~b})\right)$. Since $Q(\tilde{\mathbf{w}}) \equiv 1(\bmod 2)$, we may apply the result of the first case to conclude that there exists a representation of $\mathbb{Z}_{2}$-cosets $\sigma_{0}: \tilde{K}+\frac{1}{2} \tilde{\mathbf{w}} \rightarrow \Sigma_{g}$ for any integer $g \geq n+3$ satisfying $g \equiv Q(\mathbf{w})+7(\bmod 8)$. Let $T_{0}$ be the $n \times g$ integral matrix corresponding to $\sigma_{0}$, and define the $n \times(g+1)$ matrix $T$ as

$$
T=\left(\begin{array}{l|c}
T_{0} & \varepsilon_{a} \\
& \vdots \\
& 0
\end{array}\right),
$$

where $\varepsilon_{a} \in \mathbb{Z}_{2}^{\times}$such that $-a=\varepsilon_{a}^{2}$. Then $T$ induces a representation of $\mathbb{Z}_{2}$-cosets

$$
\sigma: K+\frac{1}{2} \mathbf{w} \rightarrow \Sigma_{g+1} \text {. }
$$

This proves the lemma since $g+1 \geq n+4$ and $g+1 \equiv Q(\mathbf{w})(\bmod 8)$.

Lemma 4.3. (1) Let $K=\mathbb{Z}\left[\mathbf{w}, \mathbf{w}^{\prime}\right]$ be a positive definite integral $\mathbb{Z}$-lattice such that $Q(\mathbf{w}) \equiv 5(\bmod 8)$ and $B\left(\mathbf{w}, \mathbf{w}^{\prime}\right) \equiv Q\left(\mathbf{w}^{\prime}\right)(\bmod 2)$. Then the $\mathbb{Z}$-coset $K+\frac{1}{2} \mathbf{w}$ is represented by $\Sigma_{5}$.

(2) Let $K=\mathbb{Z}[\mathbf{w}] \perp K^{\prime}$ be a $\mathbb{Z}$-lattice such that $Q(\mathbf{w})=6$ and $K^{\prime}$ is a positive definite even integral $\mathbb{Z}$-lattice of rank 2 . Then $K+\frac{1}{2} \mathbf{w}$ is represented by $\Sigma_{6}$.

Proof. Since the proofs for (1) and (2) are quite similar to each other, we only provide the proof of (1). For the sake of convenience, put $a=Q(\mathbf{w}), b=B\left(\mathbf{w}, \mathbf{w}^{\prime}\right)$ 
and $c=Q\left(\mathbf{w}^{\prime}\right)$. By Corollary 2.5, it is enough to show, for any prime $p$, that

$$
K_{p}+\frac{1}{2} \mathbf{w} \rightarrow\left(\Sigma_{5}\right)_{p}
$$

In case when $p \neq 2, K_{p}+\frac{1}{2} \mathbf{w}=K_{p}$ is represented by $\left(I_{5}\right)_{p}=\left(\Sigma_{5}\right)_{p}$ by Theorem 2 of [14. When $p=2$, we have, by hypothesis, that

$$
2\left(K_{2}+\mathbb{Z}_{2}\left[\frac{1}{2} \mathbf{w}\right]\right) \cong\langle a\rangle \perp\left\langle 4 a\left(a c-b^{2}\right)\right\rangle \quad \text { and } \quad 4 a\left(a c-b^{2}\right) \in 8 \mathbb{Z}_{2} .
$$

Therefore, by following the argument of the first case of the proof of Lemma 4.2 similarly, one may conclude $2\left(K_{2}+\mathbb{Z}_{2}\left[\frac{1}{2} \mathbf{w}\right]\right)$ is represented by $2\left(\left(I_{5}\right)_{2}+\mathbb{Z}_{2}\left[\frac{1}{2} \mathbf{v}_{5}\right]\right)$ so that $K_{2}+\frac{1}{2} \mathbf{w}$ is represented by $\left(\Sigma_{5}\right)_{2}$.

Let $n$ be a positive integer and let $i, j$ be integers such that $1 \leq i, j \leq n$, let $E_{i j}$ be the $n \times n$ matrix with 1 in the $(i, j)$ position and 0 elsewhere.

Lemma 4.4. Let $n \geq 3$ be a positive integer and let $n_{0}$ be an integer such that $1 \leq n_{0} \leq n$. Let $A=\operatorname{diag}\left(a_{1}, \ldots, a_{n}\right)$ be a diagonal matrix in $M_{n}(\mathbb{Z})$ and $S=\left(s_{i j}\right)$ be a symmetric matrix in $M_{n}(\mathbb{Z})$. Suppose that $A, S$ and $n_{0}$ satisfy the following conditions:

(i) $a_{i}+s_{i i} \equiv s_{i n_{0}}(\bmod 2)$ for any $i \neq n_{0}$,

(ii) $a_{i}>2 n(n-1)(3 n+2)$ for any $i$,

(iii) $a_{i} a_{j} \geq 4 n^{2}\left|s_{i j}\right|^{2}$ for any $1 \leq i \leq j \leq n$.

Then, $A+S$ is a positive definite symmetric matrix and we have

$$
K+\frac{1}{2} \mathbf{w} \rightarrow \Sigma_{6 \cdot \frac{(n-1)(n-2)}{2}+5(n-1)+k_{0}}
$$

for some integer $0 \leq k_{0} \leq 10$, where $K=\mathbb{Z}\left[\mathbf{d}_{1}, \ldots, \mathbf{d}_{n}\right]$ whose Gram matrix with respect to $\left\{\mathbf{d}_{1}, \ldots, \mathbf{d}_{n}\right\}$ is $A+S$ and $\mathbf{w}=\mathbf{d}_{n_{0}}$.

Proof. By condition (i) we can write, for each $i \neq n_{0}, a_{i}+s_{i i}=\sum_{1 \leq j \leq n, j \neq i} t_{i j}$ such that

$$
t_{i j} \equiv\left\{\begin{array}{ll}
0(\bmod 2) & \text { if } j \neq n_{0}, \\
s_{i n_{0}}(\bmod 2) & \text { if } j=n_{0},
\end{array} \quad \text { and } \quad t_{i j} \geq 2\left\lfloor\frac{a_{i}+s_{i i}-1}{2(n-1)}\right\rfloor .\right.
$$

Since $a_{i} \geq 2 n\left|s_{i i}\right|$ by condition (ii) we have

$$
a_{i}+s_{i i} \geq \frac{2 n-1}{2 n} a_{i}=\frac{n-1}{n} a_{i}+\frac{1}{2 n} a_{i} .
$$

Hence, by condition (iii), one may verify that

$$
t_{i j} \geq 2\left\lfloor\frac{a_{i}+s_{i i}-1}{2(n-1)}\right\rfloor>\frac{a_{i}+s_{i i}-1}{n-1}-2 \geq \frac{a_{i}}{n}+\frac{a_{i}-2 n(2 n-1)}{2 n(n-1)}>\frac{a_{i}}{n} .
$$

Similarly, we can write $a_{n_{0}}+s_{n_{0} n_{0}}=6 \cdot \frac{(n-1)(n-2)}{2}+\sum_{1 \leq j \leq n, j \neq n_{0}} t_{n_{0} j}+r_{0}$, where

$$
t_{n_{0} j} \equiv 5(\bmod 8), t_{n_{0} j} \geq\left\lfloor\frac{a_{n_{0}}+s_{n_{0} n_{0}}}{n-1}-3(n-2)\right\rfloor-7,
$$

and $0 \leq r_{0} \leq 7(n-1)$. One may also show that $t_{n_{0} j}>\frac{a_{n_{0}}}{n}$. Therefore, by condition (iii) we have $t_{i j} t_{j i}>\frac{a_{i} a_{j}}{n^{2}} \geq 4\left|s_{i j}\right|^{2} \geq\left|s_{i j}\right|^{2}$ for any $i \neq j$. 
Now, we can decompose $A+S$ as follows:

$$
\begin{aligned}
A+S= & \sum_{i}\left(a_{i}+s_{i i}\right) E_{i i}+\sum_{1 \leq i, j \leq n} s_{i j} E_{i j} \\
= & \sum_{\substack{i<j \\
i, j \neq n_{0}}}\left(6 E_{n_{0} n_{0}}+t_{i j} E_{i i}+t_{j i} E_{j j}+s_{i j} E_{i j}+s_{j i} E_{j i}\right) \\
& +\sum_{j \neq n_{0}}\left(t_{n_{0} j} E_{n_{0} n_{0}}+t_{j n_{0}} E_{j j}+s_{n_{0} j} E_{n_{0} j}+s_{j n_{0}} E_{j n_{0}}\right)+r_{0} E_{n_{0} n_{0}} .
\end{aligned}
$$

Hence, one may easily observe that $A+S$ is positive definite. Moreover, since $t_{i j} t_{j i}-\left|s_{i j}\right|^{2}>0$ for any $i \neq j$, we can apply Lemma 4.3 so that each

$$
\left(6 E_{n_{0} n_{0}}+t_{i j} E_{i i}+t_{j i} E_{j j}+s_{i j} E_{i j}+s_{j i} E_{j i}\right)+\frac{1}{2} \mathbf{w}
$$

is represented by $\Sigma_{6}$ for any $i<j$ with $i, j \neq i_{t}$, and for each $j \neq n_{0}$,

$$
\left(t_{n_{0} j} E_{n_{0} n_{0}}+t_{j n_{0}} E_{j j}+s_{n_{0} j} E_{n_{0} j}+s_{j n_{0}} E_{j n_{0}}\right)+\frac{1}{2} \mathbf{w}
$$

is represented by $\Sigma_{5}$. Furthermore, $r_{0} E_{n_{0} n_{0}}+\frac{1}{2} \mathbf{w}$ can be represented by $\Sigma_{k_{0}}$ for some $0 \leq k_{0} \leq 10$, for $g_{\Delta}(1)=10$. Thus,

$$
(A+S)+\frac{1}{2} \mathbf{w} \rightarrow \Sigma_{6 \cdot \frac{(n-1)(n-2)}{2}+5(n-1)+k_{0}}
$$

for some $0 \leq k_{0} \leq 10$, which proves the lemma (see Remark $3.3(\mathrm{~b})$ ).

\section{UPPER BOUND FOR $g_{\Delta}(n)$}

In this section, we will derive an upper bound for $g_{\Delta}(n)$ and complete the proof of Theorem 1.1. We begin by describing the "balanced HKZ reduction" introduced in Section 4 of [1] in terms of $\mathbb{Z}$-lattices. Let $U(n)$ be the group of upper triangular unipotent matrices in $M_{n}(\mathbb{R})$. Let $K$ be a positive definite $\mathbb{Z}$-lattices of rank $n$ and let $\left\{\mathbf{d}_{1}, \ldots, \mathbf{d}_{n}\right\}$ be a basis for $K$. We say that a basis $\left\{\mathbf{d}_{1}, \ldots, \mathbf{d}_{n}\right\}$ for $K$ is balanced HKZ-reduced if its corresponding Gram matrix $M$ is of the form $H[X]:=X^{t} H X$, where $X=\left(x_{i j}\right) \in U(n)$ and $H=\operatorname{diag}\left(h_{1}, \ldots, h_{n}\right)$ satisfy the following two properties:

(1) $h_{1}=\mu(K)$ and $h_{i} h_{j}^{-1} \leq \alpha(j-i)$ for any $1 \leq i<j \leq n$;

(2) $\left|x_{i j}\right|,\left|y_{i j}\right| \leq c(j-i)$ for any $1 \leq i \leq j \leq n$, where $X^{-1}=\left(y_{i j}\right)$.

Here, $\alpha(m):=\sigma_{m+1} \prod_{k=2}^{m+1} \sigma_{k}^{\frac{1}{k-1}}, \sigma_{k}=4 \pi^{-1} \Gamma\left(\frac{k}{2}+1\right)^{\frac{2}{k}}$ and $c(m)$ is the coefficient of $x^{m}$ in the Maclaurin series of $e^{\frac{x / 2}{1-x}}$. Note that every positive definite $\mathbb{Z}$-lattice has a "balanced HKZ-reduced" basis (see [1, Section 4]). On the other hand, we can bound the values $\alpha(j-i)([17$, Corollary 2.5]) as

$$
\alpha(j-i) \leq \bar{\alpha}(n):=e^{\ln (n+1)+(\ln (n+1))^{2}} .
$$

Furthermore, there exists an absolute constant $D \geq 1$ such that

$$
2 c(j-i) \leq \bar{c}(j-i):=D e^{\sqrt{2(j-i)}},
$$

for any $1 \leq i \leq j \leq n$ ([16], see also [9, p.547]). Note that $\bar{c}(m)$ is an increasing function of $m$. 
Proposition 5.1. Let $n \geq 3$ be an integer and let

$$
G(n):=144 D^{6} n^{12} e^{4\left(\ln (n+1)+(\ln (n+1))^{2}\right)} e^{(4+4 \sqrt{2}) \sqrt{n / 2}},
$$

where $D$ is the absolute constant in (5.2). Then every $\mathbb{Z}$-coset $K+\frac{1}{2} \mathbf{w}$ satisfying condition (i) of Lemma 4.1 can be represented by $\Sigma_{6 \cdot \frac{(n-1)(n-2)}{2}+5(n-1)+n+k_{0}}$ for some integer $0 \leq k_{0} \leq 10$, provided that $\mu(K) \geq G(n)$.

Proof. The proof of this proposition is motivated by Section 6 of [1] and a modification of the arguments in there. The strategy of the proof is outlined as follows. We will take a specific basis for $K$ whose Gram matrix will be denoted by $M$. Then we will take a diagonal matrix $A=\operatorname{diag}\left(a_{1}, \ldots, a_{n}\right)$, with all the $a_{i}$ 's as large as possible, such that $M-A$ remains positive semidefinite. Then we will take $P \in M_{n}(\mathbb{Z})$ such that $P^{t} P$ approximates $M-A$ well and $P^{t} P+\frac{1}{2} \mathbf{w}$ is represented by $\Sigma_{n}$. Write $M-A$ as $P^{t} P+S$, or equivalently, $M=P^{t} P+A+S$. We will show that $A$ and $S$ satisfy all conditions in Lemma 4.4. As a result, $(A+S)+\frac{1}{2} \mathbf{w}$ will be represented by $\Sigma_{6 \cdot \frac{(n-1)(n-2)}{2}+5(n-1)+k_{0}}$ for some $0 \leq k_{0} \leq 10$. Hence we will conclude that $M+\frac{1}{2} \mathbf{w}$ can be represented by $\Sigma_{6 \cdot \frac{(n-1)(n-2)}{2}+5(n-1)+n+k_{0}}$ for some $1 \leq k_{0} \leq 10$.

Let $\left\{\mathbf{d}_{1}, \ldots, \mathbf{d}_{n}\right\}$ be a balanced HKZ-reduced basis for $K$ whose corresponding Gram matrix is $H[X]$, where $H$ is a diagonal matrix $\operatorname{diag}\left(h_{1}, \ldots, h_{n}\right), h_{1}=\mu(K)$, and $X \in U(n)$ which satisfy (1) and (2). Let $\mathbf{w}=\mathbf{d}_{i_{1}}+\cdots+\mathbf{d}_{i_{t}}$ for some $1 \leq$ $i_{1}<\cdots<i_{t} \leq n$ and $t \geq 1$. With respect to the basis obtained by replacing $\mathbf{d}_{i_{t}}$ with $\mathbf{w}, K \cong M:=H\left[X^{\prime}\right]$ where $X^{\prime}:=X T$ and $T \in U(n)$ is defined as $T:=I_{n}+E_{i_{1} i_{t}}+\cdots+E_{i_{t-1} i_{t}}$. We note that $T^{-1}=I_{n}-\left(E_{i_{1} i_{t}}+\cdots+E_{i_{t-1} i_{t}}\right)$. If we put $X^{\prime}=\left(x_{i j}^{\prime}\right)$ and $\left(X^{\prime}\right)^{-1}=T^{-1} X^{-1}=\left(y_{i j}^{\prime}\right)$, then by a straight forward computation using (5.2) we obtain

$$
\left|x_{i j}^{\prime}\right| \leq n \bar{c}(j-i), \quad\left|y_{i j}^{\prime}\right| \leq \bar{c}(j-i) \quad \text { for any } 1 \leq i<j \leq n .
$$

Now, for any $1 \leq k \leq n$, we let

$$
a_{k}:=\left\lfloor\frac{1}{n^{2}} \bar{\alpha}(n)^{-1} \bar{c}(n-k)^{-2} h_{k}\right\rfloor,
$$

and let $A:=\operatorname{diag}\left(a_{1}, \ldots, a_{n}\right)$. Following the same argument used in the proof of Proposition 6.3 of [1], we can find an upper triangular matrix $N=\left(n_{i j}\right)$ such that

$$
I_{n}-A\left[X^{\prime-1} \sqrt{H}^{-1}\right]=N^{t} N .
$$

Note that $\left|n_{i j}\right| \leq 1$ for any $i \leq j$, since $1-\sum_{i \leq j}\left|n_{i j}\right|^{2}$ is the $(j, j)$ entry of $I_{n}-N^{t} N=A\left[X^{\prime-1} \sqrt{H}^{-1}\right]$ which is positive semidefinite.

Let $W=\left(w_{i j}\right)$ be the upper triangular matrix $N \sqrt{H} X^{\prime}$ in $M_{n}(\mathbb{R})$. Then $W^{t} W=$ $M-A$. We can take an integral matrix $P=\left(p_{i j}\right)$ satisfying

$$
\left|w_{i j}-p_{i j}\right| \leq 1 \quad \text { and } \quad p_{i i_{t}} \equiv 1(\bmod 2)
$$

for any $1 \leq i, j \leq n$. Let $Q=\left(q_{i j}\right):=W-P$. Then

$$
P^{t} P=(W-Q)^{t}(W-Q)=M-A-Q^{t} W-W^{t} Q+Q^{t} Q,
$$

hence $M=P^{t} P+A+S$, where $S=\left(s_{i j}\right):=Q^{t} W+W^{t} Q-Q^{t} Q$ which is an integral symmetric matrix. We note that $P^{t} P+\frac{1}{2} \mathbf{w}$ is represented by $\Sigma_{n}$. Therefore, as outlined at the beginning of the proof, it is enough to show that $A$ and $S$ satisfy the conditions in Lemma 4.4 with $n_{0}=i_{t}$. 
To verify the first condition, let $M=\left(m_{i j}\right)$ and note that $m_{i i} \equiv m_{i i_{t}}(\bmod 2)$ for any $i$, by the hypothesis of this proposition. Also, the $(i, i)$ and the $\left(i, i_{t}\right)$ entries of $P^{t} P$ have the same parity for any $i$, by the construction of $P$. Since $A+S=M-P^{t} P$, the first condition in Lemma 4.4 is satisfied.

Now we estimate the lower bound of $a_{i}$. By the hypothesis, we have

$$
\bar{\alpha}(n) h_{i} \geq h_{1}=\mu(K) \geq G(n)=144 D^{6} n^{12} e^{4\left(\ln (n+1)+(\ln (n+1))^{2}\right)} e^{(4+4 \sqrt{2}) \sqrt{n / 2}} .
$$

Combining this with the fact that $\bar{c}(n-j)^{2} \bar{c}(j)^{2}=D^{4} e^{2 \sqrt{2}(\sqrt{n-j}+\sqrt{j})}$ is maximized at $j=\frac{n}{2}$, we have

$$
\bar{\alpha}(n) h_{i} \geq 144 n^{12} \bar{\alpha}(n)^{4} \bar{c}(n)^{2} \bar{c}(n-j)^{2} \bar{c}(j)^{2},
$$

for any $1 \leq j \leq n$. Hence, we have $\frac{1}{n^{2}} \bar{\alpha}(n)^{-1} \bar{c}(n-i)^{-2} h_{i} \geq n^{10} \geq 1$, so that

$$
a_{i}=\left\lfloor\frac{1}{n^{2}} \bar{\alpha}(n)^{-1} \bar{c}(n-i)^{-2} h_{i}\right\rfloor \geq \frac{1}{2 n^{2}} \bar{\alpha}(n)^{-1} \bar{c}(n-i)^{-2} h_{i},
$$

and, especially $a_{i} \geq n^{10}>2 n(n-1)(3 n+2)$ for any $i$. This proves that the second condition in Lemma 4.4 is satisfied.

On the other hand, using (5.1), (5.3), and the fact that $\left|n_{i j}\right| \leq 1$, one may obtain that $\left|w_{i j}\right| \leq n^{2} \bar{c}(j)\left(\bar{\alpha}(n) h_{j}\right)^{\frac{1}{2}}$ for any $1 \leq i \leq j \leq n$. Furthermore, since $S=Q^{t} W+W^{t} Q-Q^{t} Q$ and $\left|q_{i j}\right| \leq 1$, one may show for each $1 \leq i \leq j \leq n$ that

$$
\left|s_{i j}\right| \leq 2 n^{3} \bar{c}(j)\left(\bar{\alpha}(n) h_{j}\right)^{\frac{1}{2}}+n \leq 3 n^{3} \bar{c}(j)\left(\bar{\alpha}(n) h_{j}\right)^{\frac{1}{2}} .
$$

Thus, by (5.4), (5.5), and (5.6), for any $1 \leq i \leq j \leq n$, we have

$$
\begin{aligned}
\frac{a_{i} a_{j}}{n^{2}} & \geq \frac{1}{4 n^{6}} \bar{\alpha}(n)^{-2} \bar{c}(n-i)^{-2} \bar{c}(n-j)^{-2} h_{i} h_{j} \\
& \geq 4\left|s_{i j}\right|^{2} \frac{\bar{\alpha}(n) h_{i}}{144 n^{12} \bar{c}(j)^{2} \bar{c}(n-j)^{2} \bar{c}(n-i)^{2} \bar{\alpha}(n)^{4}} \\
& \geq 4\left|s_{i j}\right|^{2} .
\end{aligned}
$$

This implies that the third condition in Lemma 4.4 is satisfied, hence we complete the proof.

Proposition 5.2. For any positive integer $n \geq 3$,

$$
g_{\Delta}(n) \leq \max \left\{g_{\Delta}(n-1)+G(n), 3 n^{2}-3 n+11\right\},
$$

where $G(n)$ is the function defined in Proposition 5.1.

Proof. Let $K+\frac{1}{2} \mathbf{w}$ be a $\mathbb{Z}$-coset in $\mathcal{K}_{n}$. If $\mu(K) \geq G(n)$, then by Proposition 5.1. $K+\frac{1}{2} \mathbf{w}$ is represented by $\Sigma_{g}$ for some integer less than or equal to

$$
6 \cdot \frac{(n-1)(n-2)}{2}+5(n-1)+n+10=3 n^{2}-3 n+11 .
$$

Suppose that $\mu(K)<G(n)$. We may assume that $K+\frac{1}{2} \mathbf{w}$ is represented by $\Sigma_{r}$ for some $r$. Furthermore we may also assume that $r \geq G(n)$. Let $K=\mathbb{Z}\left[\mathbf{d}_{1}, \ldots, \mathbf{d}_{n}\right]$, $\frac{1}{2} \mathbf{w}=w_{1} \mathbf{d}_{1}+\cdots+w_{n} \mathbf{d}_{n}$ and define

$$
f\left(x_{1}, \ldots, x_{n}\right):=4 \cdot Q\left(x_{1} \mathbf{d}_{1}+\cdots+x_{n} \mathbf{d}_{n}+\frac{1}{2} \mathbf{w}\right) .
$$


We may further assume that $\left\{\mathbf{d}_{1}, \ldots, \mathbf{d}_{n}\right\}$ is a balanced HKZ reduced basis for $K$ so that $Q\left(\mathbf{d}_{1}\right)=\mu(K)$. As is described in (3.2), there are $r$ linear forms $L_{1}\left(x_{1}, \ldots, x_{n}\right), \ldots, L_{r}\left(x_{1}, \ldots, x_{n}\right)$ over $\mathbb{Z}$ and integers $c_{1}, \ldots, c_{r}$ such that

$$
f\left(x_{1}, \ldots, x_{n}\right)=\sum_{j=1}^{r} 4 \cdot\left(L_{j}\left(x_{1}, \ldots, x_{n}\right)+c_{j}+\frac{1}{2}\right)^{2} .
$$

Since $f\left(-w_{1}, \ldots,-w_{n}\right)=0$, we have $-L_{j}\left(w_{1}, \ldots, w_{n}\right)+c_{j}+\frac{1}{2}=0$ for any $1 \leq j \leq r$. Hence, for any $1 \leq j \leq r$, we have

$$
L_{j}\left(x_{1}, \ldots, x_{n}\right)+c_{j}+\frac{1}{2}=L\left(x_{1}+w_{1}, \ldots, x_{n}+w_{n}\right) .
$$

If $b_{1}, \ldots, b_{r}$ are the coefficients of $x_{1}$ in $L_{1}, \ldots, L_{r}$ respectively, then at most $\lfloor G(n)\rfloor$ of them are nonzero. Thus, without loss of generality, we can write

$$
\begin{aligned}
f\left(x_{1}, \ldots, x_{n}\right)= & \sum_{j=1}^{\lfloor G(n)\rfloor} 4 \cdot\left(L_{j}\left(x_{1}, \ldots, x_{n}\right)+c_{j}+\frac{1}{2}\right)^{2} \\
& +\sum_{j=\lfloor G(n)\rfloor+1}^{r} 4 \cdot\left(L_{j}\left(0, x_{2}, \ldots, x_{n}\right)+c_{j}+\frac{1}{2}\right)^{2} .
\end{aligned}
$$

Note that $\left(L_{j}\left(0, x_{2}, \ldots, x_{n}\right)+c_{j}+\frac{1}{2}\right)^{2}=L_{j}\left(0, x_{2}+w_{2}, \ldots, x_{n}+w_{n}\right)^{2}$. Thus, the second sum is zero or a complete quadratic polynomial in $n-1$ variables represented by $\Delta_{r-\lfloor G(n)\rfloor}$. Hence it is represented by $\Delta_{g}$ for some integer $g \leq g_{\Delta}(n-1)$. Hence, the proposition follows immediately from this.

Proof of Theorem 1.1. Clearly, $G(n)>3 n^{2}-3 n+11$. Hence, by Proposition 5.2

$$
g_{\Delta}(n) \leq \sum_{j=3}^{n} G(j)+g_{\Delta}(2), \quad \text { for } n \geq 3 .
$$

We will show that $g_{\Delta}(2)=12$ in Section 6 . Therefore, we have

$$
g_{\Delta}(n) \leq n G(n)=144 D^{6} n^{13} e^{4\left(\ln (n+1)+(\ln (n+1))^{2}\right)} e^{(4+4 \sqrt{2}) \sqrt{n / 2}} .
$$

Since $144 D^{6} n^{13} e^{4\left(\ln (n+1)+(\ln (n+1))^{2}\right)}=O\left(e^{\varepsilon \sqrt{n}}\right)$ for any $\varepsilon>0$, we may conclude that

$$
g_{\Delta}(n)=O\left(e^{(4+2 \sqrt{2}+\varepsilon) \sqrt{n}}\right) .
$$

\section{Exact VALUe OF $g_{\Delta}(n)$ FOR $2 \leq n \leq 4$}

In this section, we always assume that $n$ is an integer such that $2 \leq n \leq 4$ and we will determine the exact value of $g_{\Delta}(n)$. Let $K+\frac{1}{2} \mathbf{w}$ be a $\mathbb{Z}$-coset in $\mathcal{K}_{n}^{*}$. From now on, we fix the following notations. We write $K=\mathbb{Z}\left[\mathbf{d}_{1}, \ldots, \mathbf{d}_{n}\right]$ and $\mathbf{w}=\mathbf{d}_{i_{1}}+\cdots+\mathbf{d}_{i_{t}}$ for some $t \geq 1$ and $1 \leq i_{1}<\cdots<i_{t} \leq n$. We denote the corresponding Gram matrix of $K$ with respect to $\left\{\mathbf{d}_{1}, \ldots, \mathbf{d}_{n}\right\}$ by $M=\left(m_{i j}\right)$ and we assume that $M$ is a Minkowski reduced symmetric matrix. By [2] (see Lemma 1.2 of page 257 ), we have

$$
0<m_{11} \leq m_{22} \leq \ldots \leq m_{n n} \text { and }\left|2 m_{i j}\right| \leq m_{i i} \forall 1 \leq i<j \leq n .
$$

We shall state two more technical lemmas, which will be used in the proof of Theorem 1.2 . 
Lemma 6.1. Let $Q(\mathbf{x})=Q\left(x_{1} \mathbf{d}_{1}+\cdots+x_{n} \mathbf{d}_{n}\right)$ be a positive definite quadratic form whose Gram matrix is a Minkowski reduced symmetric matrix $M=\left(m_{i j}\right)$. Then, for any $1 \leq i \leq n$, we have

$$
Q(\mathbf{x}) \geq C(n) m_{i i} x_{i}^{2},
$$

where $C(2)=\frac{3}{4}, C(3)=\frac{1}{2}$ and $C(4)=\frac{1}{5}$.

Proof. We only provide a proof in the case when $n=4$. Other cases can be proved similarly ( $c f$. see Lemma 2.3 of [3]). Fix an integer $i$ in $\{1,2,3,4\}$ and let $j<k<l$ be the remaining three integers listed in increasing order. Let

$$
D_{j k l}=m_{j j} m_{k k} m_{l l}-m_{j j} m_{k l}^{2}-m_{k k} m_{j k}^{2}-m_{l l} m_{j k}^{2}+2 m_{j k} m_{k l} m_{j l},
$$

which is the determinant a $3 \times 3$ submatrix of $M$. Hence, $D_{j k l}$ is positive, since $Q(\mathbf{x})$ is positive definite. From the fact that $\gamma_{4}^{4}=4$, where $\gamma_{4}$ is the 4-dimensional Hermite constant, we have $m_{11} m_{22} m_{33} m_{44} \leq 4 D$, where $D$ is the discriminant of $Q$. We refer readers to [2, Theorem 2.2, 3.1 of Chapter 12] and [18, Satz 7] for more details. By (6.1), we have

$$
D_{j k l} m_{i i} \leq\left(\frac{5}{4} m_{j j} m_{k k} m_{l l}\right) m_{i i} \leq 5 D .
$$

Now, by completing the squares, we have

$$
Q\left(x_{1} \mathbf{d}_{1}+\cdots+x_{4} \mathbf{d}_{4}\right) \geq m_{j j}\left(x_{j}+\cdots\right)^{2}+\cdots+\frac{D}{D_{j k l}} x_{i}^{2} \geq \frac{1}{5} m_{i i} x_{i}^{2} .
$$

Hence, we prove the lemma.

Lemma 6.2. Let $K+\frac{1}{2} \mathbf{w} \in \mathcal{K}_{n}^{*}$ and $Q(\mathbf{w}) \equiv k(\bmod 8)$ with $1 \leq k \leq 8$. Furthermore, let $S_{2}=\{5,6,7,8\}, S_{3}=\{6,7,8\}$ and $S_{4}=\{7,8\}$.

(1) If $Q(\mathbf{w}) \leq 8$ or $k \in S_{n}$, then $K+\frac{1}{2} \mathbf{w} \rightarrow \Sigma_{k}$.

(2) Suppose that $Q(\mathbf{w})>8$ and $k \notin S_{n}$. If there are non-negative integers $k_{i_{1}}, \ldots, k_{i_{t}}$ such that

$$
k_{i_{1}}+\cdots+k_{i_{t}}=k \quad \text { and } \quad Q\left(x_{1} \mathbf{d}_{1}+\cdots+x_{n} \mathbf{d}_{n}\right)-\left(k_{i_{1}} x_{i_{1}}^{2}+\cdots+k_{i_{t}} x_{i_{t}}^{2}\right)
$$

is a positive definite quadratic form. Then $K+\frac{1}{2} \mathbf{w} \rightarrow \Sigma_{k+8}$.

Proof. (1) If $Q(\mathbf{w}) \leq 8$ then the result follows from Lemma 4.1. Now we assume that $k \in S_{n}$. Since $k \geq n+3, K_{p}+\frac{1}{2} \mathbf{w}=K_{p}$ is represented by $\left(I_{k}\right)_{p}=\left(\Sigma_{k}\right)_{p}$ for any prime $p \neq 2$. Also, by Lemma 4.2, $K_{2}+\frac{1}{2} \mathbf{w}$ is represented by $\left(\Sigma_{k}\right)_{2}$. Thus, by Corollary 2.5, $K+\frac{1}{2} \mathbf{w} \rightarrow \Sigma_{k}$.

(2) Let $\sigma_{0}: K+\frac{1}{2} \mathbf{w} \rightarrow \Sigma_{r}$ be a representation of $\mathbb{Z}$-cosets. Consider another $\mathbb{Z}$-coset $K^{\prime}+\frac{1}{2} \mathbf{w}^{\prime}$, where $K^{\prime}=\mathbb{Z}\left[\mathbf{d}_{1}^{\prime}, \ldots, \mathbf{d}_{n}^{\prime}\right]$ is a $\mathbb{Z}$-lattice whose Gram matrix with respect to $\left\{\mathbf{d}_{1}^{\prime}, \ldots, \mathbf{d}_{n}^{\prime}\right\}$ is equal to

$$
M^{\prime}=M-\left(k_{i_{1}} E_{i_{1} i_{1}}+\cdots+k_{i_{t}} E_{i_{t} i_{t}}\right)
$$

and $\mathbf{w}^{\prime}=\mathbf{d}_{i_{1}}^{\prime}+\cdots+\mathbf{d}_{i_{t}}^{\prime}$. We note that $K^{\prime}$ is positive definite and $Q\left(\mathbf{w}^{\prime}\right)=Q(\mathbf{w})-k$ is a positive integer congruent to 0 modulo 8 by the hypothesis.

Let $T_{0}$ be the $n \times r$ integral matrix corresponding to $\sigma_{0}$ and let $\varepsilon$ be a unit in $\mathbb{Z}_{2}$ such that $-1=7 \varepsilon^{2}$. We consider the following $n \times(r+7 k)$ matrix $T^{\prime}$ over $\mathbb{Z}_{2}$ : 


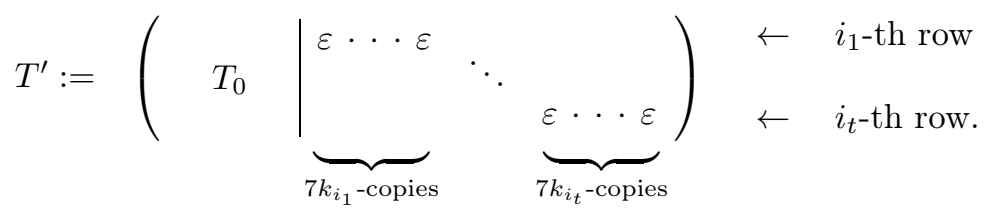

Here, $\varepsilon$ 's are all placed on $i_{a}$-th row for each $1 \leq a \leq t$, only one $\varepsilon$ is placed on each column and 0's are placed elsewhere. Then $T^{\prime}$ induces a representation $\sigma^{\prime}:\left(K^{\prime}\right)_{2}+\frac{1}{2} \mathbf{w}^{\prime} \rightarrow\left(\Sigma_{r+7 k}\right)_{2}$ (see Remark 3.3 (a)), hence by Lemma 4.2, we have

$$
\left(K^{\prime}\right)_{2}+\frac{1}{2} \mathbf{w}^{\prime} \rightarrow\left(\Sigma_{8}\right)_{2}
$$

It is clear that $K_{p}^{\prime}+\frac{1}{2} \mathbf{w}=K_{p}^{\prime}$ is represented by $\left(I_{8}\right)_{p}=\left(\Sigma_{8}\right)_{p}$ for any prime $p \neq 2$. Thus, by Corollary 2.5, there is a representation of $\mathbb{Z}$-cosets $\sigma_{1}: K^{\prime}+\frac{1}{2} \mathbf{w}^{\prime} \rightarrow \Sigma_{8}$. If we let $T_{1}$ be the $n \times 8$ matrix corresponding to $\sigma_{1}$, then the following $n \times(k+8)$ matrix $T$ over $\mathbb{Z}$

$$
T:=\left(T_{1} \mid \begin{array}{llll}
1 \cdots 1 & \ddots & \\
& & \underbrace{\cdots \cdots 1}_{k_{i_{1}} \text {-copies }}
\end{array}\right) \leftarrow i_{i_{i_{t}} \text {-copies }}{ }^{\text {-th row }},
$$

induces a representation of $\mathbb{Z}$-cosets $\sigma: K+\frac{1}{2} \mathbf{w} \rightarrow \Sigma_{k+8}$.

We are now ready to prove Theorem 1.2, First, we shall prove the following Proposition.

Proposition 6.3. We have $g_{\Delta}(n) \geq n+10$ for any $2 \leq n \leq 4$.

Proof. Let $K=\mathbb{Z}\left[\mathbf{d}_{1}, \mathbf{d}_{2}\right]$ be a $\mathbb{Z}$-lattice whose Gram matrix with respect to $\left\{\mathbf{d}_{1}, \mathbf{d}_{2}\right\}$ is $\left(\begin{array}{cc}8 & 2 \\ 2 & 12\end{array}\right)$ and $\mathbf{w}=\mathbf{d}_{2}$. If $K+\frac{1}{2} \mathbf{w}$ is represented by $\Sigma_{r}$, then $r \equiv 4(\bmod 8)$ by Lemma 4.1. Note that the following matrix

$$
T:=\left(\begin{array}{cccccccccccc}
1 & 1 & 1 & 1 & 1 & -1 & -1 & -1 & 0 & 0 & 0 & 0 \\
1 & 1 & 1 & 1 & 1 & 1 & 1 & 1 & 1 & 1 & 1 & 1
\end{array}\right)
$$

induces a representation of $\mathbb{Z}$-cosets $K+\frac{1}{2} \mathbf{w} \rightarrow \Sigma_{12}$ (see Remark 3.3 (a)). However, $K+\frac{1}{2} \mathbf{w}$ cannot be represented by $\Sigma_{4}$, since $K$ cannot be represented by $I_{4}$ over $\mathbb{Q}_{2}$. Thus, we have $g_{\Delta}(2) \geq 12$.

For the case when $n=3$ or 4 , we consider a $\mathbb{Z}$-lattice $K=\mathbb{Z}\left[\mathbf{d}_{1}, \ldots, \mathbf{d}_{n}\right]$ whose Gram matrix with respect to $\left\{\mathbf{d}_{1}, \ldots, \mathbf{d}_{n}\right\}$ is a diagonal matrix

$$
\operatorname{diag}(3,3,23) \text { or } \operatorname{diag}(1,3,3,23) \text {, respectively, }
$$

and $\mathbf{w}=\sum_{i=1}^{n} \mathbf{d}_{i}$. Then $Q(\mathbf{w}) \equiv 5$ or $6(\bmod 8)$, respectively, and one may find a representation of $\mathbb{Z}$-cosets from $K+\frac{1}{2} \mathbf{w}$ to $\Sigma_{13}$ or $\Sigma_{14}$, respectively. However, $K$ cannot be represented by $I_{5}$ or $I_{6}$, respectively, over $\mathbb{Q}_{3}$. Hence, we have $g_{\Delta}(3) \geq 13$ and $g_{\Delta}(4) \geq 14$.

Proof of Theorem 1.2. By Propositions 3.4 and 6.3, it is enough to prove that $g_{\Delta}(n) \leq n+10$ for each $2 \leq n \leq 4$. The proof is a case-by-case analysis according to $n$ and the shape of $\mathbf{w}$. For each case, the proof will show how we can determine $g_{\Delta}(n) \leq n+10$. 
We assume that $Q(\mathbf{w}) \equiv k(\bmod 8)$ with $1 \leq k \leq 8$ and let $r_{K, \mathbf{w}}$ be the integer defined in Lemma 4.1. Also, we assume that the Gram matrix $M$ of $K$ is Minkowski reduced so that $M$ satisfies all conditions given in (6.1). Also, by replacing $\mathbf{d}_{j}$ with $\pm \mathbf{d}_{j}$ suitably, we may further assume that

$$
m_{1 j} \geq 0 \text { for any } 2 \leq j \leq n .
$$

Under the conditions (6.1) and (6.2), the necessary and sufficient condition for $M$ to be a Minkowski reduced positive definite form is that

$$
m_{i j} \geq-\frac{1}{2}\left(m_{11}+m_{i i}\right)+m_{1 i}+m_{1 j} \quad \text { for any } 2 \leq i<j \leq n,
$$

and when $n=4$,

$$
\begin{aligned}
&-\frac{1}{2}\left(m_{22}+m_{33}\right)-m_{23}-m_{24}, \\
& m_{34} \geq \begin{array}{l}
2 \\
-
\end{array}\left(m_{22}+m_{33}\right)+m_{23}+m_{24}, \\
&-\frac{1}{2}\left(m_{11}+m_{22}+m_{33}\right)+m_{12}+m_{13}+m_{14}-m_{23}-m_{24}, \\
&-\frac{1}{2}\left(m_{11}+m_{22}+m_{33}\right)-m_{12}+m_{13}+m_{14}+m_{23}+m_{24}, \\
& \\
& \frac{1}{2}\left(m_{22}+m_{33}\right)-m_{23}+m_{24}, \\
& m_{34} \leq \frac{1}{2}\left(m_{22}+m_{33}\right)+m_{23}-m_{24}, \\
& \frac{1}{2}\left(m_{11}+m_{22}+m_{33}\right)-m_{12}+m_{13}-m_{14}-m_{23}+m_{24}, \\
& \frac{1}{2}\left(m_{11}+m_{22}+m_{33}\right)-m_{12}-m_{13}+m_{14}+m_{23}-m_{24} .
\end{aligned}
$$

(Case 1) We shall prove $g_{\Delta}(2) \leq 12$ by showing $K+\frac{1}{2} \mathbf{w}$ is represented by $\Sigma_{r}$ for some $r \leq 12$. By Lemma 4.1 and part (1) of Lemma 6.2, we may assume that condition (i) of Lemma 4.1 holds and

$$
Q(\mathbf{w})>12, r_{K, \mathbf{w}}>12 \text { and } 1 \leq k \leq 4 .
$$

Case 1-(i) Assume that $\mathbf{w}=\mathbf{d}_{i}$ for $i=1$ or 2 . From the assumption, we have $\overline{m_{i i}>12}$. Hence, we have $Q(\mathbf{x})-k x_{i}^{2}$ is positive definite, since by Lemma 6.1

$$
Q(\mathbf{x})-k x_{i}^{2} \geq \frac{3}{4} \max \left(m_{11} x_{1}^{2}, m_{22} x_{2}^{2}\right)-4 x_{i}^{2}>0,
$$

for any $\mathbf{x} \neq \mathbf{0}$. Therefore, by Lemma 6.2 (2), we conclude that $K+\frac{1}{2} \mathbf{w}$ is represented by $\Sigma_{k+8}$, where $k+8 \leq 12$.

Case 1-(ii) Assume that $\mathbf{w}=\mathbf{d}_{1}+\mathbf{d}_{2}$. If $m_{22} \geq 6$, then $Q(\mathbf{x})-k x_{2}^{2}$ is positive definite by Lemma 6.1, hence we are done by Lemma 6.2. For any $M$ satisfying $1 \leq m_{11} \leq m_{22} \leq 5$ and $0 \leq m_{12} \leq \frac{1}{2} m_{11}$, it does not satisfy the assumption of (Case 1). This proves Case 1 .

(Case 2) Now we shall prove $g_{\Delta}(3) \leq 13$ by showing $K+\frac{1}{2} \mathbf{w}$ is represented by $\Sigma_{r}$ for some $r \leq 13$. As in Case 1, we may assume that condition (i) of Lemma 4.1 holds and

$$
Q(\mathbf{w})>13, r_{K, \mathbf{w}}>13 \text { and } 1 \leq k \leq 5 .
$$

Case 2-(i) Assume that $\mathbf{w}=\mathbf{d}_{i}$ for $i=1,2$ or 3. From the assumption, we have $\overline{m_{i i}}>13$. Hence, we have $Q(\mathbf{x})-k x_{i}^{2}$ is positive definite, since by Lemma 6.1

$$
Q(\mathbf{x})-k x_{i}^{2} \geq \frac{1}{2} \max \left(m_{11} x_{1}^{2}, m_{22} x_{2}^{2}, m_{33} x_{3}^{2}\right)-5 x_{i}^{2}>0,
$$


for any $\mathbf{x} \neq \mathbf{0}$. Therefore, by Lemma 6.2 (2), we conclude that $K+\frac{1}{2} \mathbf{w}$ is represented by $\Sigma_{k+8}$, where $k+8 \leq 13$.

Case 2-(ii) Assume that $\mathbf{w}=\mathbf{d}_{1}+\mathbf{d}_{2}$. If $m_{22} \geq 10$ then $Q(\mathbf{x})-k x_{2}^{2}$ is positive definite by Lemma 6.1, hence we are done by Lemma 6.2(2). Now, we may assume that

$$
1 \leq m_{11} \leq m_{22} \leq 9 \quad \text { and } \quad 0 \leq m_{12}, m_{13} \leq \frac{1}{2} m_{11}
$$

We note that for each triple $\left(m_{11}, m_{22}, m_{12}\right)$ satisfying the assumption of Case 2, there exist non-negative integers $k_{1}, k_{2}$ such that $k_{1}+k_{2}=k$ and $Q\left(x_{1} \mathbf{d}_{1}+x_{2} \mathbf{d}_{2}\right)-$ $\left(k_{1} x_{1}^{2}+k_{2} x_{2}^{2}\right)$ is positive definite. Once $m_{11}, m_{22}, m_{12}, m_{13}$ are decided, there are only finitely many candidates of $m_{23}$ by (6.2) and (6.3).

Now, for each fixed $\left(m_{11}, m_{22}, m_{12}, m_{13}, m_{23}\right)$, we do the following process. Let $m_{33}$ be the smallest integer greater than or equal to $m_{22}$ satisfying condition (i) of Lemma 4.1. We search for non-negative integers $k_{1}, k_{2}$ such that $k_{1}+k_{2}=k$ and $Q(\mathbf{x})-\left(k_{1} x_{1}^{2}+k_{2} x_{2}^{2}\right)$ is positive definite. Once we find such $k_{1}, k_{2}$, then we are done by Lemma 6.2 (2). Otherwise, we put the matrix $M$ in a list, raise $m_{33}$ by 2 and then repeat searching for $k_{1}, k_{2}$. Note that this process ends in a finite number of steps, since the discriminant of the form $Q(\mathbf{x})-\left(k_{1} x_{1}^{2}+k_{2} x_{2}^{2}\right)$ is an increasing linear function of $m_{33}$ for each possible pair $\left(k_{1}, k_{2}\right)$. Running this process by a computer program, the final list of matrices obtained is empty.

Case 2-(iii) For the remaining cases, we have $i_{t}=3$. If $m_{33} \geq 10$, then $Q(\mathbf{x})-k x_{3}^{2}$ is positive definite by Lemma 6.1 Thus, we are done by Lemma 6.2 (2). Hence, we are left with finitely many candidates of $M$ all of which have $m_{33} \leq 9$. For each such $M$ satisfying the assumption of Case 2, by a computer program, we can find non-negative integers $k_{i_{1}}, \ldots, k_{i_{t}}$ such that

$$
k_{i_{1}}+\cdots+k_{i_{t}}=k \text { and } Q(\mathbf{x})-\left(k_{i_{1}} x_{i_{1}}^{2}+\cdots+k_{i_{t}} x_{i_{t}}^{2}\right)
$$

is positive definite. Thus, we are done by Lemma 6.2 (2).

(Case 3) Lastly, we shall prove $g_{\Delta}(4) \leq 14$ by showing $K+\frac{1}{2} \mathbf{w}$ is represented by $\Sigma_{r}$ for some $r \leq 14$. As before, we may assume that condition (i) of Lemma 4.1 holds and

$$
Q(\mathbf{w})>14, r_{K, \mathbf{w}}>14 \text { and } 1 \leq k \leq 6 .
$$

Case 3-(i) Assume that $i_{t}=4$, where there are 8 possible cases. If $m_{44} \geq 31$, then by Lemma 6.1 $Q(\mathbf{x})-k x_{4}^{2}$ is positive definite, since

$$
Q(\mathbf{x})-k x_{4}^{2} \geq \frac{1}{5} \max \left(m_{11} x_{1}^{2}, m_{22} x_{2}^{2}, m_{33} x_{3}^{2}, m_{44} x_{4}^{2}\right)-6 x_{4}^{2}>0,
$$

for any $\mathbf{x} \neq \mathbf{0}$. Thus, we are done by Lemma 6.2 (2). By (6.1)-6.5), we are left with finitely many candidates of $M$ to check. For each of these $M$ that satisfies the assumption of Case 3, by a computer program, we can find non-negative integers $k_{i_{1}}, \ldots, k_{i_{t}}$ such that

$$
k_{i_{1}}+\cdots+k_{i_{t}}=k \quad \text { and } \quad Q(\mathbf{x})-\left(k_{i_{1}} x_{i_{1}}^{2}+\cdots+k_{i_{t}} x_{i_{t}}^{2}\right)
$$


is positive definite, except for the four $\mathbb{Z}$-cosets $M+\frac{1}{2} \mathbf{w}$, where $\mathbf{w}=\mathbf{d}_{1}+\mathbf{d}_{4}$ and $M$ is one of the following matrices:

$$
\left(\begin{array}{cccc}
9 & 3 & 3 & 2 \\
3 & 9 & 3 & -4 \\
3 & 3 & 9 & -4 \\
2 & -4 & -4 & 9
\end{array}\right),\left(\begin{array}{cccc}
9 & 3 & 4 & 2 \\
3 & 9 & 3 & -4 \\
4 & 3 & 9 & -3 \\
2 & -4 & -3 & 9
\end{array}\right),\left(\begin{array}{cccc}
9 & 4 & 3 & 2 \\
4 & 9 & 3 & -3 \\
3 & 3 & 9 & -4 \\
2 & -3 & -4 & 9
\end{array}\right),\left(\begin{array}{cccc}
9 & 4 & 4 & 2 \\
4 & 9 & 3 & -3 \\
4 & 3 & 9 & -3 \\
2 & -3 & -3 & 9
\end{array}\right) .
$$

Note that, two $\mathbb{Z}$-cosets corresponding to the first matrix and the last one with $\mathbf{w}=\mathbf{d}_{1}+\mathbf{d}_{4}$ are isometric to each other, and they are represented by $\Sigma_{14}$. Also, the other two matrices also give an equivalent $\mathbb{Z}$-cosets, which are represented by $\Sigma_{14}$. This, together with Lemma 6.2 (2), implies the claim in this case.

Case 3-(ii) Assume that $i_{t}=3$, where there are 4 possible cases. If $m_{33} \geq 31$, then $\overline{Q(\mathbf{x})-k x_{3}^{2}}$ is positive definite by Lemma 6.1 Thus, we are done by Lemma 6.2 (2). Now, we may assume that

$$
1 \leq m_{11} \leq m_{22} \leq m_{33} \leq 30 .
$$

Then there are only finitely many candidates of matrix $M-m_{44} E_{44}$. Note that, for each candidate that we should concern, there exist non-negative integers $k_{i_{1}}, \ldots, k_{i_{t}}$ such that

$$
k_{i_{1}}+\cdots+k_{i_{t}}=k \text { and } Q\left(x_{1} \mathbf{d}_{1}+x_{2} \mathbf{d}_{2}+x_{3} \mathbf{d}_{3}\right)-\left(k_{i_{1}} x_{i_{1}}^{2}+\cdots+k_{i_{t}} x_{i_{t}}^{2}\right)
$$

is positive definite. We can run a process similar to the one described in the Case 2-(ii). However this time we have four matrices on the final list and they appear when $\mathbf{w}=\mathbf{d}_{1}+\mathbf{d}_{3}$. Each of the corresponding $\mathbb{Z}$-cosets is isometric to one of the $\mathbb{Z}$-cosets described in Case 3-(i). Hence we proves the claim.

Case 3-(iii) Assume that $i_{t}=2$. By a similar argument as before, we may assume that $1 \leq m_{11} \leq m_{22} \leq 30$. Then there are only finitely many candidates of 7 -tuple $\left(m_{11}, m_{12}, m_{13}, m_{14}, m_{22}, m_{23}, m_{24}\right)$. For each of these 7 -tuples that satisfies the assumption of Case 3, we can check that $Q\left(x_{1} \mathbf{d}_{1}+x_{2} \mathbf{d}_{2}\right)-k x_{2}^{2}$ is positive definite and $C:=m_{11}-\frac{5 m_{12}^{2}}{2\left(m_{22}-k\right)}>0$. Let $m_{22}^{\prime}=m_{22}-k$ and put

$$
C_{0}:=\max \left(\frac{10}{3} \cdot \frac{m_{23}}{m_{22}^{\prime}}+\frac{m_{13}\left(m_{13}+m_{14}\right)}{C}, \frac{10}{3} \cdot \frac{m_{24}}{m_{22}^{\prime}}+\frac{m_{14}\left(m_{13}+m_{14}\right)}{C}\right) .
$$

If $m_{33}>\frac{8}{3} C_{0}$, then $Q(\mathbf{x})-k x_{2}^{2}$ is greater than or equal to

$$
\begin{array}{r}
\frac{2 m_{22}^{\prime}}{5}\left(x_{2}+\frac{5}{2} \frac{m_{12}}{m_{22}^{\prime}} x_{1}\right)^{2}+\frac{3 m_{22}^{\prime}}{10}\left(x_{2}+\frac{10}{3} \frac{m_{13}}{m_{22}^{\prime}} x_{3}\right)^{2}+\frac{3 m_{22}^{\prime}}{10}\left(x_{2}+\frac{10}{3} \frac{m_{14}}{m_{22}^{\prime}} x_{4}\right)^{2} \\
+C \cdot \frac{m_{13}}{m_{13}+m_{14}}\left(x_{1}+\frac{m_{13}}{C} x_{3}\right)^{2}+C \cdot \frac{m_{14}}{m_{13}+m_{14}}\left(x_{1}+\frac{m_{14}}{C} x_{4}\right)^{2} \\
+Q\left(x_{3} \mathbf{d}_{3}+x_{4} \mathbf{d}_{4}\right)-C_{0}\left(x_{3}^{2}+x_{4}^{2}\right) .
\end{array}
$$

Since $m_{44} \geq m_{33}>\frac{8}{3} C_{0}$ and $Q\left(x_{3} \mathbf{d}_{3}+x_{4} \mathbf{d}_{4}\right) \geq \frac{3}{8}\left(m_{33} x_{3}^{2}+m_{44} x_{4}^{2}\right)$ by Lemma 6.1 we conclude that $Q(\mathbf{x})-k x_{2}^{2}$ is positive definite. Hence, we are done by Lemma $6.2(2)$.

Now, we may assume that $m_{33} \leq \frac{8}{3} C_{0}$, so that there are only finitely many candidates of matrix $M-m_{44} E_{44}$. We note that the quadratic form $Q\left(x_{1} \mathbf{d}_{1}+x_{2} \mathbf{d}_{2}+\right.$ $\left.x_{3} \mathbf{d}_{3}\right)-k x_{2}^{2}$ is positive definite for each of these candidates. We run the same 
process as described in the Case 3-(ii) and obtain a list of four matrices $M$ which appear only when $\mathbf{w}=\mathbf{d}_{1}+\mathbf{d}_{2}$. Each of the corresponding $\mathbb{Z}$-cosets is isometric to one of the $\mathbb{Z}$-cosets described in Case 3-(i). Hence we proves the claim.

Case 3-(iv) Finally, we assume that $\mathbf{w}=\mathbf{d}_{1}$. One may check by Lemma 6.1 that $\overline{Q(\mathbf{x})-k x_{1}^{2}}$ is positive definite except for $m_{11} \in\{20,21,22,30\}$. Hence, by Lemma 6.2 (2), we may assume that $m_{11} \in\{20,21,22,30\}$, so that there are only finitely many candidates of 4 -tuple $\left(m_{11}, m_{12}, m_{13}, m_{14}\right)$.

Put $m_{11}^{\prime}=m_{11}-k$ and $m_{234}=m_{12}+m_{13}+m_{14}$. If $m_{234}=0$, that is, $m_{12}=m_{13}=m_{14}=0$, then $Q(\mathbf{x})-k x_{1}^{2}$ is positive definite obviously. Otherwise, we note that

$$
\begin{aligned}
Q(\mathbf{x})-k x_{1}^{2}= & \sum_{j=2}^{4} m_{11}^{\prime} \frac{m_{12}}{m_{234}}\left(x_{1}+\frac{m_{234}}{m_{11}^{\prime}} x_{2}\right)^{2} \\
& +Q\left(x_{2} \mathbf{d}_{2}+x_{3} \mathbf{d}_{3}+x_{4} \mathbf{d}_{4}\right)-\frac{m_{234}}{m_{11}^{\prime}} \cdot \sum_{j=2}^{4} m_{1 j} x_{j}^{2} .
\end{aligned}
$$

Hence, if $m_{22}>2 \cdot \frac{m_{234}^{2}}{m_{11}^{\prime}}$, then $Q(\mathbf{x})-k x_{1}^{2}$ is positive definite, since

$$
Q\left(x_{2} \mathbf{d}_{2}+x_{3} \mathbf{d}_{3}+x_{4} \mathbf{d}_{4}\right) \geq \sum_{j=2}^{4} \frac{1}{2} \frac{m_{1 j}}{m_{234}} m_{j j} x_{j}^{2}>\frac{m_{234}}{m_{11}^{\prime}} \cdot \sum_{j=2}^{4} m_{1 j} x_{j}^{2},
$$

by Lemma 6.1. Thus, we may assume that $m_{22} \leq 2 \cdot \frac{m_{234}^{2}}{m_{11}^{\prime}}$ and there are only finitely many candidates of 7 -tuple $\left(m_{11}, m_{12}, m_{13}, m_{14}, m_{22}, m_{23}, m_{24}\right)$. By a similar argument used in Case 3-(iii), we may further assume that $m_{33}$ is bounded and run the process as described in the other cases. This time the final list is empty and so we are done.

Remark 6.4. One may naturally expect that $g_{\Delta}(5)=15$. However, if we consider a $\mathbb{Z}$-coset $K+\frac{1}{2} \mathbf{d}_{5}$, where $K=\mathbb{Z}\left[\mathbf{d}_{1}, \ldots, \mathbf{d}_{5}\right]$ whose Gram matrix with respect to $\left\{\mathbf{d}_{1}, \ldots, \mathbf{d}_{5}\right\}$ is a diagonal matrix $\operatorname{diag}(2,2,2,2,16)$, then one may verify that

$$
K+\frac{1}{2} \mathbf{d}_{5} \rightarrow \Sigma_{16}, \quad \text { but } K+\frac{1}{2} \mathbf{d}_{5} \nrightarrow \Sigma_{8}
$$

which implies that $g_{\Delta}(5) \geq 16$.

Acknowledgments. I would like to express my gratitude to Professor ByeongKweon Oh who is my supervisor and Professor Wai Kiu Chan for their valuable advice, and to the referee for carefully reading this paper and making many helpful comments.

\section{REFERENCES}

[1] C. N. Beli, W. K. Chan, M. I. Icaza and J. Liu, On a Waring's problem for integral quadratic and hermitian forms, Tran. Amer. Math. Soc., electronically published on September 28, 2018, DOI : https://doi.org/10.1090/tran/7571 (to appear in print).

[2] J. W. S. Cassels, Rational quadratic forms, Academic Press, London, 1978.

[3] W. K. Chan and B.-K. Oh, Representations of integral quadratic polynomials, Contemp. Math. 587 (2013), 31-46.

[4] J. S. Hsia, Y. Kitaoka and M. Kneser, Representations of positive definite quadratic forms, J. Reine Angew. Math. 301 (1978), 132-141.

[5] M. I. Icaza, Sums of squares of integral linear forms, Acta Arith. 124 (1996), 231-241. 
[6] K. Kim and B.-K. Oh, A sum of squares not divisible by a prime, arXiv:1805.03038, 2018

[7] M.-H. Kim and B.-K. Oh, Representations of positive definite senary integral quadratic forms by a sum of squares, J. Number Theory 63 (1997), 89-100.

[8] M.-H. Kim and B.-K. Oh, Representations of integral quadratic forms by sums of squares, Math. Z. 250 (2005), 427-442.

[9] K. Knopp, Theory and application of infinite series, Blackie \& Son, Ltd., London and Glasgow, 1951.

[10] C. Ko, On the representation of a quadratic form as a sum of squares of linear forms, Quart. J. Math. Oxford 8 (1937), 81-98.

[11] C. Ko, On the decomposition of quadratic forms in six variables, Acta Arith. 3 (1939), 64-78.

[12] L. J. Mordell, A new Waring's problem with squares of linear forms, Quart. J. Math. Oxford 1 (1930), 276-288.

[13] L. J. Mordell, The representation of a definite quadratic form as a sum of two others, Ann. of Math. (2) 38 (1937), 751-757.

[14] O. T. O'Meara, The integral representations of quadratic forms over local field, Amer. J. Math. 80 (1958), 843-878

[15] O. T. O'Meara, Introduction to quadratic forms, Springer Verlarg, New York, 1963.

[16] O. Perron, Über das Verhalten einer ausgearteten hypergeometrischen Reihe bei unbegrenztem Wachstum eines Parameters, J. Reine Angew. Math. 151 (1921), 63-78.

[17] C. P. Schnorr, A hierarchy of polynomial time lattice basis reduction algorithms, Theoret. Comput. Sci. 53 (1987), 201-224.

[18] L. van der Waeden, Die Reduktionstheorie der positiven quadratischen Formen, Acta Math. 96 (1956), 265-309.

Department of Mathematical Sciences, Seoul National University, Seoul 151-747, Korea

E-mail address: goodkdj@snu.ac.kr 